\title{
Religious Involvement, Inflammatory Markers and Stress Hormones in Major Depression and Chronic Medical Illness
}

\author{
Denise L. Bellinger'1, Lee S. Berk ${ }^{1,2,3}$, Harold G. Koenig,4,5,6*, Noha Daher ${ }^{2,7}$ \\ Michelle J. Pearce ${ }^{3,4,8}$, Clive J. Robins ${ }^{4,9}$, Bruce Nelson ${ }^{10}$, Sally F. Shaw ${ }^{10}$, \\ Harvey Jay Cohen ${ }^{3,5}$, Michael B. King11 \\ ${ }^{1}$ Department of Pathology and Human Anatomy, School of Medicine, Loma Linda University, Loma Linda, CA, \\ USA \\ ${ }^{2}$ Allied Health Studies, School of Allied Health Professions, Loma Linda University, Loma Linda, CA, USA \\ ${ }^{3}$ Center for Spirituality, Theology and Health, Duke University, Durham, NC, USA \\ ${ }^{4}$ Department of Psychiatry and Behavioral Sciences, Duke University Medical Center, Durham, NC, USA \\ ${ }^{5}$ Department of Medicine, Duke University Medical Center, Durham, NC, USA \\ ${ }^{6}$ Department of Medicine, King Abdulaziz University, Jeddah, Saudi Arabia \\ ${ }^{7}$ Epidemiology, Biostatistics and Population Medicine, School of Public Health, Loma Linda University, \\ Loma Linda, CA, USA \\ ${ }^{8}$ School of Medicine, University of Maryland, Baltimore, USA \\ ${ }^{9}$ Department of Psychology and Neuroscience, Duke University Medical Center, Durham, NC, USA \\ ${ }^{10}$ Department of Research, Glendale Adventist Medical Center, Glendale, CA, USA \\ ${ }^{11}$ Division of Psychiatry, Faculty of Brain Sciences, University College, London, UK \\ Email: $\underline{\text { Harold.Koenig@duke.edu }}$
}

Received 17 July 2014; revised 10 August 2014; accepted 8 September 2014

Copyright (C) 2014 by authors and Scientific Research Publishing Inc.

This work is licensed under the Creative Commons Attribution International License (CC BY).

http://creativecommons.org/licenses/by/4.0/

(c) (i) Open Access

\section{Abstract}

Background: Religious practices/experiences (RPE) may produce positive physiological changes in patients with major depressive disorder (MDD) and chronic medical illness. Here, we report cross-sectional relationships between depressive symptoms, RPE and stress biomarkers (pro-/ anti-inflammatory measures and stress hormones), hypothesizing positive associations between depressive symptoms and stress biomarkers and inverse associations between RPE and stress biomarkers. Methods: We recruited 132 individuals with both MDD and chronic illness into a randomized clinical trial. First, stress biomarkers in the baseline sample were compared to biomarker levels from a community sample. Second, relationships between depressive symptoms and biomarkers were examined, and, finally, relationships between RPE and biomarkers were

*Corresponding author.

How to cite this paper: Bellinger, D.L., Berk, L.S., Koenig, H.G., Pearce, N.D.M.J., Robins, C.J., Nelson, B., Shaw, S.F., Cohen, H.J. and King, M.B. (2014) Religious Involvement, Inflammatory Markers and Stress Hormones in Major Depression and Chronic Medical Illness. Open Journal of Psychiatry, 4, 335-352. http://dx.doi.org/10.4236/ojpsych.2014.44040 
analyzed, controlling for demographics, depressive symptoms, and physical functioning. Results: As expected, inflammatory markers and stress hormones were higher in our sample with MDD compared to community participants. In the current sample, however, depressive symptoms were largely unrelated to stress biomarkers, and were unexpectedly inversely related to proinflammatory cytokine levels (TNF- $\alpha$, IL-1 $\beta$ ). Likewise, while RPE were largely unrelated to stress biomarkers, they were related to the anti-inflammatory cytokine IL-1RA and the stress hormone norepinephrine in expected directions. Unexpectedly, RPE were also positively related to the proinflammatory cytokine IFN- $\gamma$ and to IFN- $\gamma /$ IL-4 and IFN- $\gamma /$ IL-10 ratios. Conclusions: Little evidence was found for a consistent pattern of relationships between depressive symptoms or religiosity and stress biomarkers. Of the few significant relationships, unexpected findings predominated. Future research is needed to determine whether religious interventions can alter stress biomarkers over time in MDD.

\section{Keywords}

\section{Religiosity, Depression, Inflammation, Immune Function, Stress Hormones}

\section{Introduction}

Depressive disorder and religious involvement are common in persons with chronic medical illness. Depressive disorder has been reported in $19 \%$ to $45 \%$ of those with chronic illness depending on the setting and diagnostic method [1]-[3], and physical disability is one of the strongest predictors of depressive symptoms in the general population [4]-[6]. Religious involvement is important to many individuals, especially those with chronic illness, since religion is often used to cope with the life changes caused by health problems. While over half of healthy persons in the U.S. indicate that religion is an important part of daily life [7] [8], the importance of religion increases even further among those with medical illness. In some areas of the country, up to $90 \%$ of medically ill persons report that they depend on religion to cope with, with over $40 \%$ saying that it is the most important factor that keeps them going [9].

Depression is also often accompanied by physiological changes that can adversely affect the course of medical illness over time, including increased levels of pro-inflammatory cytokines, decreased levels of anti-inflammatory cytokines, and increased stress hormones. Alterations in immune and endocrine function associated with depression may adversely affect health by increasing risk of infection [10], inflammatory disorders [11], and even malignancy [12]-[14]. The etiological relationship between depression and these physiological changes, however, is a complex one that is likely bi-directional in nature [15]. In fact, depression is known to stimulate some components of the immune system and suppress others. Furthermore, certain immune elements (such as pro-inflammatory cytokines) can lead to sickness behaviors that resemble depression, which has led to the possibility that altered immune and endocrine functions may be etiologically related to depression, especially when it develops in a setting of chronic stress [16].

Regardless of direction of effect, major depressive disorder has been associated with a host of immune [17], endocrine [18], and inflammatory measures [19], including an altered balance in the T-helper (Th)1/Th2 cytokine ratio, i.e., higher levels of pro-inflammatory Th1 cytokines (IL-6, IL-12, IFN- $\gamma$ ) and monocytic cytokines (IL-1, IL-6, TNF- $\alpha$ ) [20]-[22] and lower levels of anti-inflammatory Th2 cytokines (IL-4, IL-10) [23] [24]. Importantly, these altered physiological functions associated with depression have been shown to normalize in response to treatment with electroconvulsive therapy (TNF- $\alpha$ ), [25] antidepressant drug therapy (TNF- $\alpha$, C-reactive protein, IL-1 $\beta$, IL-6) [26]-[28], and psychological interventions (IL1-RA, IL-6, IFN- $\gamma$ ) [29]. Coping behaviors and psychological interventions that increase positive emotions and neutralize negative ones may also be effective in reducing pro-inflammatory markers such as IL-6 [30] [31], TNF- $\alpha$ [31], C-reactive protein (CRP) [31], and IFN- $\gamma$ [31], and in normalizing catecholamine [31] and cortisol levels [32]-[33].

Religious beliefs and practices may prevent the development of depression, promote the resolution of depression, and/or help persons with depressive disorder cope with the illness [34]-[36]. Given the physiological alterations that occur in depression, religious beliefs and behaviors may help to normalize those changes. In fact, a 
number of studies have reported lower levels of pro-inflammatory markers (IL-6) [37]-[39] and stress hormones (specifically cortisol) among those who are more religious [40]-[44]. Furthermore, spiritual interventions have been shown to reduce the pro-/anti-inflammatory cytokine ratios [45] [46], reduce cortisol [47]-[51], and decrease catecholamine [52] [53] levels.

\section{Hypotheses}

The present report examines cross-sectional relationships between religious involvement, depressive symptoms, indicators of inflammation, and stress hormones in persons with major depressive disorder and chronic medical illness. We hypothesize that:

1) Pro-inflammatory cytokines (CRP, IL-6) and stress hormone (cortisol, epinephrine, norepinephrine) in our sample with major depressive disorder will be higher than in a community-dwelling sample drawn from the Midlife in the United States (MIDUS) Survey;

2) After adjustment for demographic factors and physical functioning, depressive symptom severity will be greater among those with high levels of pro-inflammatory cytokines, high pro-inflammatory/anti-inflammatory cytokine ratios, and high stress hormone levels; as a specific hypothesis, depressive symptom severity will be greater among those with high levels of CRP, IL-6, and urinary cortisol;

3) After adjustment for demographics, depressive symptoms, and physical functioning, religious practices and experiences will be lower among those with high levels of pro-inflammatory cytokines, high pro-inflammatory/ anti-inflammatory cytokine ratios, and high stress hormone levels, as a specific hypothesis, overall religiosity will be lower among those with high serum CRP, serum IL-6, and urinary cortisol.

\section{Methods}

\subsection{Procedures}

Participants aged 18 to 85 were recruited into a randomized clinical trial conducted at two sites, one in Durham, North Carolina, and the other in Los Angeles County, California. Inclusion criteria were: 1) the presence of at least one chronic medical condition for 6 months or longer; 2) a DSM-IV diagnosis of major depressive disorder; 3) mild to moderately severe depressive symptoms (10 to 40 on the Beck Depression Inventory); and 4) religion or spirituality at least somewhat important (since the clinical trial involved a religious intervention). Exclusion criteria were: 1) significant cognitive impairment; 2) receipt of psychotherapy within the past two months; 3) a diagnosis of psychotic disorder, substance abuse, or posttraumatic stress disorder (PTSD) within the past year; 4) bipolar disorder; 5) active suicidal thoughts; and 6) human immunodeficiency virus/acquired immunodeficiency syndrome (HIV/AIDS), autoimmune diseases, dementia, endocrine disorders, a prognosis of less than 6 months, or taking immunosuppressant drugs. Study coordinators screened potential participants by telephone and then arranged a face-to-face interview, when written informed consent was obtained. Those who met the inclusion criteria were then enrolled into the study and completed a baseline evaluation. The Duke University Medical Center institutional review board (protocol \#26533) and Glendale Adventist Medical Center (3/17/11) approved the study.

\subsection{Measures}

Physical and mental. The 12-item Duke Activity Status Index (DASI) [54] was used to measure physical functioning across domains for physical and instrumental activities of daily living, with higher scores indicating better physical function (range 12 - 36). Cognitive functioning was assessed by the brief Mini-Mental State Exam [55], which measures cognitive function on a range from 0 to 18, with significant cognitive impairment defined as scores below 15. Major depressive disorder was diagnosed using corresponding modules of the Mini-International Neuropsychiatric Interview (MINI) [56], a structured psychiatric interview that follows standard DSMIV criteria. Depression severity was assessed using the Beck Depression Inventory (BDI-II) [57]. The BDI is a 21-item self-report depression inventory with scores ranging from 0 to 63, and is a most widely used instrument for assessing depressive symptoms in primary care settings. Diagnoses of psychotic disorder, alcohol abuse, drug abuse or PTSD within the past year, lifetime history of bipolar disorder, and presence of active suicidal thoughts were determined using corresponding modules of the MINI.

Religious. Multiple domains of RPE were assessed including denomination, self-rated religiosity, public and 
private religious activity, intrinsic religiosity, daily spiritual experiences, and religious coping. To determine study eligibility, a single item was used to measure self-rated religiosity/spirituality and asked, "How important is religion/spirituality in your daily life?" (those indicating "not important” were excluded). Two items from the Duke University Religiosity Index [58] were used to measure public and private religious activity. For public religious activity (adapted to chronically ill disabled patients), participants were asked, "When you were physically able, how often did you attend religious services or other religious meetings?” (responses ranged from "never" [1] to "more than once a week" [6]). For private religious activity, participants were asked: "How often do you spend time in private religious activities, such as prayer, meditation or scripture study?" (responses ranged from "rarely or never" [1] to "more than once a day" [6]). Intrinsic religious motivation was assessed using a validated 10-item intrinsic religiosity scale that has been used in both community and medical populations (range 10 - 50) [59]. Religious and spiritual experiences were examined using the 16-item Daily Spiritual Experiences Scale [60] (range 16 - 94). Finally, religious coping was measured using the 14-item Brief RCOPE [61], which is made up of two 7-item subscales assessing positive (PRC) and negative religious coping (NRC) (0 - 21 range for each subscale). We reverse scored items on the NRC subscale and combined them with PRC scores to form an overall religious coping measure (range 0 - 42). To increase the power of the analysis for the primary hypotheses, religious variables were combined into an overall religiosity measure by summing public and private religious activity, daily spiritual experiences, intrinsic religiosity, and religious coping. In order to provide equal weighting for each religiosity variable, given varied metrics, we rescaled each measure to a 0 - 1 scale and summed them to create an overall religiosity variable (range 0 to 5 ) (alpha $=0.88$ ).

Demographic. Demographic variables included age, gender, race, and education. Age and education (years of education) were left as continuous, whereas gender was categorized as female (1) vs. male (0) and race as white (1) vs. non-white (Black, Hispanic, Asian) (0).

Biomarkers. Serum was collected from venous blood collected in serum separator Vaccutainer tubes. Levels of serum inflammatory markers (TNF- $\alpha$, IFN- $\gamma$, IL-1 $\beta$, IL-4, IL-6, IL-10, IL-12(p70)) were measured using Millipore's multiplexed high sensitivity cytokine magnetic bead-based immunoassay kits (Milliplex cat \#HSTCMAG-28SK, EMD Millipore, Billerica, MA) according to the manufacturer's instructions. IL-1RA was run using the Milliplex Human Cytokine kit (Hcytomag-60K according to manufacturer's instruction with the following manufacturer's recommendations to increase sensitivity: sample volume was doubled, from $25 \mu$ to $50 \mu \mathrm{l}$ and an additional standard dilution was added. The mean fluorescence intensity values were then divided by two in the data analyses to adjust for greater sample volume. Minimal detectable levels in $\mathrm{pg} / \mathrm{ml}$ were 0.15 for TNF- $\alpha, 0.32$ for IFN- $\gamma, 0.12$ for IL-1 $\beta$, 0.2 for IL-1RA, 1.24 for IL-4, 0.13 for IL-6, 0.58 for IL-10, and 0.15 for IL-12(p70). Intra- and inter -assay coefficient of variance (CV) were $<6 \%$ and $<20 \%$ for all cytokines, respectively. Plates were read on the MAGPIX (Luminex Corp., Austin, TX), and the data analyzed on MasterPlex 2010 (Hitachi Solutions America, San Bruno, CA). All samples were run in duplicate. Samples were repeated if the CV between the duplicates was greater than 15\%. Pro-inflammatory-to-anti-inflammatory ratios were calculated from mean values.

Serum CRP was measured using enzyme-linked immunosorbent assay (ELISA) kits from Assaypro, St. Charles, MO), which had a minimal detection of $\sim 0.25 \mathrm{ng} / \mathrm{ml}$ and an intra- and inter-assay CV of $5.0 \%$ and 7.1\%, respectively. Cortisol concentrations were determined in 12-h urine samples using ELISA kits (Enzo Life Sciences International Inc., Plymouth Meeting, PA) that had a lower limit of detection of $333 \mathrm{pg} / \mathrm{ml}$, and intraand inter-assay CV of $10.5 \%$ and $13.4 \%$, respectively. Cortisol levels were normalized for urine volume using creatinine levels determined by parametric kits that employ the Jaffe reaction (R\&D Systems, Minneapolis, MN; minimal detection of $0.01 \mathrm{mg} / \mathrm{dL}$ and intra- and inter-assay CV of $3.5 \%$ and $4.0 \%$, respectively). Plates were read using a plate reader ( $\mu$ Quant, Biotek Instruments, Inc., Winooski, VT) set at the appropriate wavelength. All samples were run in duplicate along with duplicate standards that were used to generate a standard curve. The amount of analyte in the unknown samples was calculated from the standard curve, and expressed as a mean \pm SEM of the two samples in $\mathrm{pg}$ or $\mathrm{mg} / \mathrm{ml}$ as appropriate. If the coefficient of variance between the duplicates was greater than $15 \%$, then another aliquot of the sample was thawed, and the assay repeated for that sample. Samples were assayed in batches to further limit any potential of inter-assay variability.

Twelve-hour urinary catecholamines (epinephrine and norepinephrine) were determined by high performance liquid chromatography with coulochem detection (HPLC-CD). All samples were run in batches along with standards and quality control samples (Biorad Lyphocheck I and II; City, State). Urine samples (1.0 ml) containing $1.0 \mathrm{ml}$ of phosphate buffer $(\mathrm{pH} 7.0), 1.0 \mathrm{ml}$ of $1.5 \mathrm{M}(\mathrm{pH}$ 8.6) Tris buffer and $200 \mu \mathrm{l} 3,4-$ 
dihydroxybenzylamine (DHBA; internal standard) were vortexed. Next, $50 \mathrm{mg}$ of acid washed alumina was added, and the tubes were agitated for $15 \mathrm{~min}$. The samples were centrifuged for $1 \mathrm{~min}$ at $200 \mathrm{rpm}$, and liquid in the samples was aspirated. The samples were washed with $1 \mathrm{ml}$ nanopure $\mathrm{H}_{2} \mathrm{O}$ three times and centrifuged after each aspiration to settle the alumina. To elute the catecholamines, $200 \mu \mathrm{l}$ of $0.1 \mathrm{M} \mathrm{HClO}_{4}$ were added to each sample, and the samples were vortexed. The tubes were centrifuged for $5 \mathrm{~min}$ at $5000 \mathrm{rpm}$, and the filtrates were collected. Samples were placed in an ESA Model 542 autosampler with a Model 582 isocratic pump with a 3 um Atlantis T3 $4.5 \mathrm{~mm} \times 150 \mathrm{~mm}$ column (Waters, Canton, Massachusetts) and detected using a CouloChem III set at $+200 \mathrm{mV}$ and $-200 \mathrm{mV}$. The mobile phase was delivered at a flow rate of $1.0 \mathrm{ml} / \mathrm{min}$. Urinary catecholamine were analyzed using EZChrom Elite Software (Scientific Software Inc., Pleasanton, CA). Concentrations were determined based on standards of known concentrations (200 ng/ml) of norepinephrine, epinephrine, and dopamine and expressed as $\mu \mathrm{g} / \mathrm{g}$ creatinine to normalize for urine volume.

\subsection{MIDUS Sample}

In order to determine how the ranges of biomarkers in the present study compared to those obtained in a community-dwelling sample of largely healthy individuals, we identified a subsample of 572 participants from Project 4 of the MIDUS II biomarker study [62]. These participants were drawn from the overall dataset $(\mathrm{n}=2500)$ by including those with at least one chronic medical illness who had available biomarker data, but excluding twins and other family members to avoid participants who were related. Participants completed the 20-item Center for Epidemiological Studies Depression (CES-D), whose scores were available for comparison with participants in our study (assessed by BDI). The presence of chronic medical illness was determined by the presence of self-reported or physician-diagnosed circulation problems, blood clots, heart murmur, TIA/stroke, anemia or other blood disease, hypercholesterolemia, diabetes, asthma, chronic obstructive pulmonary disease or emphysema, tuberculosis, thyroid disease, peptic ulcer disease, cancer, arthritis, glaucoma, cirrhosis/liver disease, or other medical condition. The study was approved by the Institutional Review Board at each of the participating MIDUS centers, and informed written consent was obtained for all participants [63]. Biological samples were collected from participants of MIDUS II after they had completed the telephone and mail surveys during a 2-day clinic visit. A 12-h urine sample was collected the day before the first clinic visit, and fasting blood samples were drawn in the morning of day 2 (see Dienberg et al. for more details [64]).

Serum IL-6 levels from the MIDUS II study were determined using Quantikine ${ }^{\circledR}$ high-sensitivity ELISA kits (R\&D Systems, Minneapolis, MN) with a reference range of $0.45-9.96 \mathrm{pg} / \mathrm{ml}$, and intra- and inter-assay CV of 4.1\% and 13\%, respectively [65]. Serum CRP levels were measured using the Behring Nephelometer II (BNII) Analyzer (Dade Behring) using an immunonepholometric assay specific for CRP (Seimens Healthcare Diagnostics, Deer-242 field, IL), which provides equivalent results to the high-sensitivity ELISA kits for CRP [66]. Urinary cortisol levels were measured using an enzymatic colorimetric assay and liquid chromatography-tandem spectrometry (LC-MS/MS). Samples were spiked with ${ }^{3} \mathrm{H}$-cortisol as an internal standard. After extraction by on-line turbulent flow HPLC, samples were analyzed using LC-MS/MS with multiple-reaction monitoring in positive mode. Inter-assay CV was $5.7 \%-8.8 \%$, intra-assay CV was $4.7 \%-5.0 \%$, and the reference range was $29.1-63.4 \mathrm{ng} / \mathrm{ml}[67]$.

\subsection{Statistical Analysis}

Descriptive statistics were used to describe the characteristics of the sample and visually compare the median and range values of inflammatory markers and stress hormones in our sample with those obtained in the MIDUS study (Table 1). Because inflammatory markers and stress hormone levels in our sample were non-normally distributed (and attempts to transform the data were not successful in achieving normality), we dichotomized levels into the bottom two-thirds (0) and top one-third (1) for analyses; values were excluded from analyses if they exceeded five standard deviations above the median. In bivariate analyses, the average level of depressive symptoms, each religious measure and overall religiosity were compared between participants with low and high levels of cytokines and stress hormones using the Student T-test (Table 2). Logistic regression was used to examine the relationship between depressive symptoms and biomarkers, controlling for demographic factors and physical functioning (DASI) (Table 3). Logistic regression was also used to examine relationships between religious measures and biomarkers, controlling for demographic factors, physical functioning, and depressive symptoms (Table 4). Given the highly exploratory nature of these analyses and relatively small sample size 
Table 1. Characteristics of current sample compared to MIDUS dataset.

\begin{tabular}{|c|c|c|}
\hline & Current & MIDUS \\
\hline & $(\mathrm{N}=132)^{1}$ & $(\mathrm{~N}=570)^{2}$ \\
\hline Demographics & $\%(N)$ Mean $(S D)$ & $\%(N)$ Mean $(S D)$ \\
\hline Age, years & $51.6(13.5)$ & $59.2(11.7)$ \\
\hline Education, years & $15.1(3.3)$ & $14.8(2.7)$ \\
\hline Gender (\% women) & $68.9(91)$ & 53.7 (307) \\
\hline Race (\% white) & $53.0(70)$ & $92.0(526)$ \\
\hline \multicolumn{3}{|l|}{ Depression } \\
\hline Beck Depression Inventory & $25.3(8.5)$ & --- \\
\hline CES-D & & $9.0(10.3)$ \\
\hline Pro-inflammatory cytokines & Median (range) & Median (range) \\
\hline $\mathrm{CRP}(\mathrm{ng} / \mathrm{ml})^{3}$ & $4.4(0.2-38.1)$ & $1.3(0.1-61.7)$ \\
\hline $\mathrm{TNF}-\alpha(\mathrm{pg} / \mathrm{ml})$ & $6.3(2.4-33.2)$ & --- \\
\hline $\mathrm{IL}-1 \beta(\mathrm{pg} / \mathrm{ml})$ & $1.1(0.01-10.3)$ & --- \\
\hline $\mathrm{IFN}-\gamma(\mathrm{pg} / \mathrm{ml})$ & $7.3(0.03-49.7)$ & --- \\
\hline IL-6 (pg/ml) & $2.1(0.01-12.7)$ & $2.1(0.2-23.0)$ \\
\hline IL-12(p70) (pg/ml) & $2.5(0.01-25.4)$ & --- \\
\hline \multicolumn{3}{|l|}{ Anti-inflammatory cytokines } \\
\hline IL-1RA (pg/ml) & $10.1(0.2-454.2)$ & --- \\
\hline IL-4 (pg/ml) & $9.7(0.01-98.5)$ & --- \\
\hline $\mathrm{IL}-10(\mathrm{pg} / \mathrm{ml})$ & $6.2(0.02-86.5)$ & --- \\
\hline \multicolumn{3}{|c|}{ Cytokine ratios (pro-/anti-inflammatory) } \\
\hline TNF- $\alpha / \mathrm{IL}-4$ & $0.6(0.08-818.0)$ & --- \\
\hline TNF- $\alpha /$ IL-10 & $1.1(0.07-260.0)$ & --- \\
\hline IL-1 $\beta /$ IL-1RA & $0.1(0.0-30.1)$ & --- \\
\hline IL-1 $\beta / \mathrm{IL}-10$ & $0.2(0.0-16.0)$ & --- \\
\hline IL- $1 \beta / \mathrm{IL}-4$ & $0.1(0.0-52.9)$ & --- \\
\hline IL-6/IL-10 & $0.3(0.0-92.0)$ & --- \\
\hline IL-6/IL-4 & $0.2(0.0-40.0)$ & --- \\
\hline IFN- $\gamma / \mathrm{IL}-4$ & $0.8(0.01-450.0)$ & --- \\
\hline IFN- $\gamma / \mathrm{IL}-10$ & $1.3(0.01-245.0)$ & --- \\
\hline IL-12/IL-10 & $0.4(0.0-46.0)$ & --- \\
\hline IL-12/IL-4 & $0.2(0.0-74.0)$ & --- \\
\hline \multicolumn{3}{|l|}{$\underline{\text { Stress hormones }}$} \\
\hline CORT (mg/L creatinine) & $30.2(1.3-136.0)$ & $11.0(0.4-212.0)$ \\
\hline EPI (mg/L creatinine) & $5.4(0.0-131.4)$ & $1.8(0.3-10.6)$ \\
\hline NE (mg/L creatinine) & $39.5(3.6-320.1)$ & $26.0(3.5-187.1)$ \\
\hline
\end{tabular}

MIDUS $=$ Midlife in the United States Study, CES-D $=$ Center for Epidemiologic Studies Depression, CRP $=$ C-reactive protein, TNF- $\alpha=$ tumor necrosis factor- $\alpha, \mathrm{IL}=$ interleukin, IFN $=$ interferon, CORT = cortisol, EPI = epinephrine, NE = norepinephrine, DA = dopamine, "--."“ = no comparison available, ${ }^{1} \mathrm{n}=122-132,{ }^{2} \mathrm{n}=561-570,{ }^{3} \mathrm{C}$-reactive protein is a "positive" acute phase protein.

limiting power, p values were not reduced as would ordinarily be appropriate for the multiple comparisons made here. Statistical analyses were done using SAS (version 9.3, SAS Institute Inc., Cary, North Carolina).

\section{Results}

Participants. Between June 2011 and June 2013, a total of 450 participants were screened by telephone for eligibility. Of those, 187 underwent in-person screening and 132 were enrolled in the study. Three subjects were included who did not fulfill inclusion/exclusion criteria but were randomized in the trial, and these were included 
Table 2. Comparison of depressive symptoms and religious indicators across high vs. low levels of inflammatory markers and stress hormones.

\begin{tabular}{|c|c|c|c|c|c|c|c|c|}
\hline & & $\begin{array}{l}\text { Depressive } \\
\text { symptoms }\end{array}$ & $\begin{array}{l}\text { Religious } \\
\text { attendance }\end{array}$ & $\begin{array}{l}\text { Private } \\
\text { prayer }\end{array}$ & $\begin{array}{l}\text { Intrinsic } \\
\text { religiosity }\end{array}$ & $\begin{array}{c}\text { Spiritual } \\
\text { experiences }\end{array}$ & $\begin{array}{l}\text { Religious } \\
\text { coping }\end{array}$ & $\begin{array}{l}\text { Overall } \\
\text { religiosity }\end{array}$ \\
\hline & & Mean (SD) & Mean (SD) & Mean $(S D)$ & Mean (SD) & Mean (SD) & Mean $(S D)$ & Mean (SD) \\
\hline \multicolumn{9}{|c|}{ Pro-inflammatory cytokines } \\
\hline \multirow[t]{2}{*}{ CRP } & Low $(n=86)$ & $25.8(8.3)$ & $3.9(1.6)$ & $3.7(1.7)$ & $35.1(8.7)$ & $57.8(16.1)$ & $29.1(6.3)$ & $3.0(1.0)$ \\
\hline & High $(n=43)$ & $24.2(8.7)$ & $3.7(1.6)$ & $3.5(1.7)$ & $34.5(7.8)$ & $57.0(16.2)$ & $29.6(6.1)$ & $2.9(1.0)$ \\
\hline \multirow[t]{2}{*}{ TNF- $\alpha$} & Low $(n=84)$ & $26.5(8.4)$ & $4.0(1.6)^{\#}$ & $3.7(1.6)$ & $35.4(8.3)$ & $57.7(16.3)$ & $29.4(6.4)$ & $3.0(1.0)$ \\
\hline & High $(\mathrm{n}=42)$ & $22.9(8.6)^{*}$ & 3.5 (1.6) & 3.5 (1.9) & 34.1 (7.9) & $57.6(15.6)$ & $29.5(5.7)$ & $2.8(1.0)$ \\
\hline \multirow[t]{2}{*}{$\mathrm{IL}-1 \beta$} & Low $(n=84)$ & $26.3(8.8)$ & $3.8(1.6)$ & $3.7(1.6)$ & $34.3(8.5)$ & $57.7(16.0)$ & $29.7(6.3)$ & $2.9(1.0)$ \\
\hline & High $(n=42)$ & $23.4(7.9)^{\#}$ & $4.0(1.6)$ & 3.5 (1.8) & $36.2(7.2)$ & 57.6 (16.3) & $28.8(6.0)$ & $3.0(1.0)$ \\
\hline \multirow[t]{2}{*}{ IFN- $\gamma$} & Low $(n=84)$ & $25.0(9.1)$ & $3.8(1.5)$ & $3.5(1.7)$ & $34.1(8.2)^{\#}$ & $55.7(15.9)^{*}$ & $28.5(6.3)^{* *}$ & $2.8(1.0)^{\#}$ \\
\hline & High $(n=42)$ & $25.8(7.5)$ & $4.0(1.7)$ & $3.8(1.6)$ & 36.7 (7.7) & $61.6(15.7)$ & $31.3(5.4)$ & $3.2(1.0)$ \\
\hline \multirow[t]{2}{*}{ IL-6 } & Low $(n=83)$ & $26.0(8.7)$ & $4.0(1.6)$ & 3.7 (1.6) & 34.8 (8.8) & $57.1(16.0)$ & $29.4(6.2)$ & $3.0(1.0)$ \\
\hline & High $(n=43)$ & $24.0(8.3)$ & $3.6(1.6)$ & 3.4 (1.9) & 35.2 (6.8) & $58.7(16.2)$ & $29.4(6.2)$ & $2.9(1.0)$ \\
\hline \multirow[t]{2}{*}{ IL-12(p70) } & Low $(n=84)$ & $26.1(8.7)$ & $3.8(1.6)$ & $3.6(1.7)$ & 35.1 (8.6) & $56.1(16.8)$ & $28.8(6.4)$ & $2.9(1.0)$ \\
\hline & High $(n=42)$ & $23.7(8.4)$ & $3.9(1.7)$ & $3.7(1.7)$ & $34.8(7.1)$ & 60.7 (13.9) & 30.7 (5.6) & $3.0(0.9)$ \\
\hline \multicolumn{9}{|c|}{ Anti-inflammatory cytokines } \\
\hline \multirow[t]{2}{*}{ IL-1RA } & Low $(n=85)$ & $25.8(8.8)$ & $3.8(1.7)$ & $3.4(1.7)$ & $33.5(8.5)^{* *}$ & $56.3(16.4)$ & $29.1(6.5)$ & $2.8(1.0)^{\#}$ \\
\hline & High $(n=42)$ & $24.1(7.5)$ & $4.0(1.4)$ & $3.9(1.6)$ & $37.5(7.8)$ & $60.4(15.3)$ & $29.8(5.8)$ & $3.2(0.9)$ \\
\hline \multirow[t]{2}{*}{ IL-4 } & Low $(n=84)$ & $25.7(8.8)$ & $3.9(1.6)$ & $3.7(1.6)$ & $34.4(8.4)$ & $56.8(15.7)$ & $29.4(6.2)$ & $3.0(1.0)$ \\
\hline & High $(n=42)$ & $24.3(8.2)$ & $3.8(1.6)$ & $3.5(1.7)$ & $36.2(7.5)$ & $60.4(15.9)$ & $29.5(6.2)$ & $2.9(0.9)$ \\
\hline \multirow[t]{2}{*}{ IL-10 } & Low $(n=83)$ & $25.6(8.5)$ & $3.9(1.5)$ & $3.8(1.6)$ & $35.1(8.1)$ & $58.3(15.6)$ & $29.8(6.4)$ & $3.0(0.9)$ \\
\hline & High $(n=42)$ & $25.0(8.9)$ & 3.7 (1.8) & $3.4(1.8)$ & 34.8 (8.3) & $57.0(17.0)$ & $28.8(5.8)$ & $2.9(1.1)$ \\
\hline \multicolumn{9}{|c|}{ Cytokine ratios (pro-/anti-inflammatory) } \\
\hline \multirow[t]{2}{*}{ TNF- $\alpha / \mathrm{IL}-4$} & Low $(n=83)$ & $25.8(8.8)$ & $3.9(1.7)$ & $3.5(1.7)$ & $35.3(8.5)$ & $57.9(16.9)$ & $29.4(6.5)$ & $2.9(1.1)$ \\
\hline & High $(n=42)$ & $24.2(8.4)$ & $3.8(1.4)$ & $4.0(1.6)$ & $34.5(7.3)$ & $57.9(13.8)$ & $29.6(5.5)$ & $3.0(0.8)$ \\
\hline \multirow[t]{2}{*}{ TNF- $\alpha / \mathrm{IL}-10$} & Low $(n=83)$ & 25.7 (8.6) & $4.1(1.6)^{*}$ & $3.5(1.7)$ & 35.2 (8.5) & $57.7(16.4)$ & $29.4(6.4)$ & $3.0(1.0)$ \\
\hline & High $(n=41)$ & $25.0(8.7)$ & $3.4(1.5)$ & $4.0(1.5)$ & $34.6(7.5)$ & $57.9(15.6)$ & $29.6(5.7)$ & $2.9(0.9)$ \\
\hline \multirow[t]{2}{*}{ IL- $1 \beta / \mathrm{IL}-1 \mathrm{RA}$} & Low $(n=81)$ & $25.4(9.0)$ & $3.7(1.6)^{\#}$ & $3.6(1.6)$ & $35.5(8.1)$ & $57.9(15.4)$ & $29.6(6.0)$ & $2.9(1.0)$ \\
\hline & High $(\mathrm{n}=41)$ & $24.8(7.7)$ & $4.2(1.6)$ & $3.6(1.8)$ & $34.0(8.2)$ & $57.2(17.6)$ & $29.2(6.7)$ & $3.0(1.0)$ \\
\hline \multirow[t]{2}{*}{$\mathrm{IL}-1 \beta / \mathrm{IL}-10$} & Low $(n=83)$ & $25.3(9.0)$ & $3.9(1.6)$ & $3.5(1.7)$ & $34.9(8.3)$ & $58.0(16.1)$ & $29.4(6.5)$ & $2.9(1.0)$ \\
\hline & High $(n=41)$ & $25.6(7.7)$ & $3.8(1.7)$ & $3.9(1.6)$ & $35.2(8.0)$ & $57.3(16.2)$ & $29.7(5.5)$ & $3.0(1.0)$ \\
\hline \multicolumn{9}{|c|}{ Cytokine ratios (pro-/anti-inflammatory) } \\
\hline \multirow[t]{2}{*}{ IL-1 $\beta / \mathrm{IL}-4$} & Low $(n=83)$ & $25.3(8.7)$ & $3.9(1.6)$ & $3.5(1.7)$ & $35.9(8.2)$ & $59.0(16.0)$ & $29.8(6.3)$ & $3.0(1.0)$ \\
\hline & High $(n=42)$ & $25.2(8.7)$ & $3.9(1.6)$ & $3.8(1.5)$ & 33.4 (7.9) & $56.0(15.4)$ & $28.9(5.8)$ & $2.9(0.9)$ \\
\hline
\end{tabular}




\begin{tabular}{|c|c|c|c|c|c|c|c|c|}
\hline \multicolumn{9}{|l|}{ Continued } \\
\hline \multirow[t]{2}{*}{ IL-6/IL-10 } & Low $(\mathrm{n}=83)$ & $25.4(9.1)$ & $4.0(1.6)$ & $3.5(1.7)$ & 34.9 (8.7) & $57.1(16.6)$ & $29.2(6.3)$ & $2.9(1.0$ \\
\hline & High $(n=41)$ & $25.6(7.6)$ & 3.7 (1.6) & $3.9(1.7)$ & $35.1(7.1)$ & $59.2(15.1)$ & $30.0(5.9)$ & $3.0(0.9)$ \\
\hline \multirow[t]{2}{*}{ IL-6/IL-4 } & Low $(n=83)$ & $25.4(9.0)$ & $3.8(1.6)$ & $3.4(1.7)^{*}$ & $34.9(8.3)$ & $56.8(16.7)$ & $29.3(6.4)$ & $2.9(1.0)$ \\
\hline & $\operatorname{High}(n=42)$ & $25.0(8.1)$ & $4.0(1.5)$ & $4.1(1.5)$ & $35.2(7.9)$ & $60.0(14.0)$ & $29.9(5.6)$ & $3.1(0.9)$ \\
\hline \multirow[t]{2}{*}{ IFN- $\gamma / \mathrm{IL}-4$} & Low $(n=83)$ & $25.0(8.9)$ & 3.7 (1.7) & $3.4(1.8)^{*}$ & $34.8(8.5)$ & $57.1(17.0)$ & $29.1(6.3)$ & $2.9(1.1)$ \\
\hline & $\operatorname{High}(n=42)$ & $25.7(8.3)$ & $4.1(1.5)$ & $4.0(1.3)$ & $35.5(7.4)$ & 59.5 (13.3) & $30.3(5.7)$ & $3.1(0.8)$ \\
\hline \multirow[t]{2}{*}{ IFN- $\gamma / \operatorname{IL} 10$} & Low $(n=83)$ & $25.2(8.8)$ & $3.8(1.7)$ & 3.5 (1.8) & $34.0(8.7)^{*}$ & $55.9(16.5)^{\#}$ & $28.3(6.3)^{* *}$ & $2.8(1.0)^{\#}$ \\
\hline & $\operatorname{High}(\mathrm{n}=41)$ & $26.0(8.1)$ & $4.0(1.5)$ & 3.9 (1.5) & $37.1(6.6)$ & $61.5(14.9)$ & $31.9(5.2)$ & $3.2(0.8)$ \\
\hline \multirow[t]{2}{*}{ IL-12/IL-10 } & Low $(\mathrm{n}=83)$ & 25.5 (8.8) & 3.9 (1.6) & 3.7 (1.7) & 34.6 (8.6) & $57.0(16.6)$ & $28.7(6.4)^{\#}$ & $2.9(1.0)$ \\
\hline & $\operatorname{High}(\mathrm{n}=41)$ & $25.2(8.3)$ & 3.8 (1.6) & 3.7 (1.7) & 35.9 (7.3) & $59.3(15.2)$ & $31.0(5.5)$ & $3.0(0.9)$ \\
\hline \multirow[t]{2}{*}{ IL-12/IL-4 } & Low $(n=83)$ & $25.5(8.8)$ & $3.8(1.7)$ & $3.4(1.7)^{*}$ & $35.0(8.6)$ & $56.9(16.8)$ & $29.1(6.4)$ & $2.9(1.0)$ \\
\hline & High $(n=42)$ & $24.9(8.5)$ & $4.0(1.5)$ & $4.1(1.5)$ & 35.2 (7.3) & 59.8 (13.7) & $30.3(5.6)$ & $3.1(0.9)$ \\
\hline \multicolumn{9}{|c|}{ Stress hormones } \\
\hline \multirow[t]{2}{*}{ CORT } & Low $(n=85)$ & $26.2(8.5)^{\#}$ & $3.8(1.6)$ & 3.7 (1.7) & $35.3(8.4)$ & $56.8(16.4)$ & $29.4(6.0)$ & $3.0(1.0)$ \\
\hline & High $(n=42)$ & $23.3(8.1)$ & $4.2(1.5)$ & $3.6(1.5)$ & $34.6(8.4)$ & $60.6(13.9)$ & $29.3(6.5)$ & $3.0(0.9)$ \\
\hline \multirow[t]{2}{*}{ EPI } & Low $(\mathrm{n}=84)$ & $26.0(8.3)$ & $4.1(1.6)$ & 3.7 (1.7) & $35.0(9.0)$ & 56.7 (16.6) & $29.1(6.4)$ & $3.0(1.1)$ \\
\hline & High $(n=42)$ & 23.5 (8.9) & $3.6(1.6)$ & $3.4(1.5)$ & $34.8(7.2)$ & $60.0(14.1)$ & 29.8 (5.8) & $2.9(0.8)$ \\
\hline \multirow[t]{2}{*}{$\mathrm{NE}$} & Low $(\mathrm{n}=84)$ & $25.4(8.4)$ & $4.0(1.6)$ & 3.7 (1.7) & 35.7 (8.3) & $57.8(16.0)$ & $29.7(6.2)$ & $3.0(1.0)$ \\
\hline & High $(\mathrm{n}=42)$ & $24.9(8.6)$ & $3.6(1.6)$ & 3.5 (1.5) & 33.2 (8.3) & $57.8(15.7)$ & $28.8(6.1)$ & $2.8(0.9)$ \\
\hline
\end{tabular}

${ }^{\#} 0.05<\mathrm{p}<0.10,{ }^{*} \mathrm{p} \leq 0.05,{ }^{* *} \mathrm{p} \leq 0.01$ (student t-test). High $=$ top one-third of biomarker variable $(\mathrm{n}=43-45)$; Low $=$ bottom two-thirds of biomarker variable $(\mathrm{n}=82$ - 87). CRP=C-reactive protein, TNF- $\alpha=$ tumor necrosis factor $\alpha$, IL = interleukin, IFN = interferon, CORT $=$ cortisol, EPI $=$ epinephrine, $\mathrm{NE}=$ norepinephrine.

Table 3. Logistic regression models examining depressive symptoms (Beck Depression Inventory) as a predictor of high levels of inflammation and stress hormones (dependent variables).

\begin{tabular}{ll}
\hline & Depressive symptoms \\
\cline { 2 - 2 } & \\
\hline Pro-inflammatory cytokines & OR $(95 \%$ CI $)$ \\
\hline CRP & $0.97(0.93-1.02)$ \\
TNF- $\alpha$ & $0.95(0.90-0.99)^{*}$ \\
IL-1 $\beta$ & $0.95(0.91-1.00)^{*}$ \\
IFN- $\gamma$ & $1.01(0.96-1.05)$ \\
IL-6 & $0.96(0.92-1.01)$ \\
IL-12 & $0.96(0.92-1.01)$ \\
\hline Anti-inflammatory cytokines & \\
\hline IL-1RA & $0.98(0.94-1.03)$ \\
IL-4 & $0.98(0.94-1.03)$ \\
IL-10 & $0.99(0.95-1.04)$ \\
\hline
\end{tabular}




\section{Continued}

\begin{tabular}{ll}
\hline Cytokine ratios (pro-/anti-inflammatory) & \\
\hline TNF- $\alpha /$ IL-4 & $0.97(0.93-1.02)$ \\
TNF- $\alpha /$ IL-10 & $1.00(0.96-1.05)$ \\
IL-1 $\beta /$ IL-1RA & $0.99(0.94-1.03)$ \\
IL-1 $\beta /$ IL-10 & $1.01(0.96-1.05)$ \\
IL-1 $\beta /$ IL-4 & $0.99(0.95-1.04)$ \\
IL-6/IL-10 & $1.00(0.96-1.05)$ \\
IL-6/IL-4 & $0.99(0.95-1.04)$ \\
IFN- $\gamma /$ IL-4 & $1.01(0.97-1.06)$ \\
IFN- $\gamma /$ IL10 & $1.02(0.97-1.07)$ \\
IL-12/IL-10 & $1.00(0.96-1.05)$ \\
IL-12/IL-4 & $0.99(0.95-1.04)$ \\
\hline Stress hormones & \\
\hline CORT & $0.96(0.92-1.01)$ \\
EPI & $0.97(0.92-1.02)$ \\
NE & $0.99(0.95-1.04)$ \\
\hline
\end{tabular}

OR = odds ratio, $\mathrm{CI}=$ confidence interval, Biomarkers dichotomized: high $=$ top $1 / 3$, low $=$ bottom $2 / 3$, * $\mathrm{p} \leq 0.05$; $\mathrm{n}=122$ - 129, Controlled for age, education, gender, race, physical functioning (Duke Activity Status Index).

Table 4. Logistic regression models examining religious characteristics as predictors of high levels of inflammatory markers and stress hormones (dependent variables).

\begin{tabular}{|c|c|c|c|c|c|c|}
\hline & Attendance & Private prayer & $\begin{array}{l}\text { Intrinsic } \\
\text { religiosity }\end{array}$ & $\begin{array}{c}\text { Spiritual } \\
\text { experiences }\end{array}$ & $\begin{array}{l}\text { Religious } \\
\text { coping }\end{array}$ & $\begin{array}{l}\text { Overall } \\
\text { religiosity }\end{array}$ \\
\hline Pro-Inflammatory & OR $(95 \% C I)$ & OR $(95 \% C I)$ & OR $(95 \% C I)$ & OR $(95 \% C I)$ & OR $(95 \% C I)$ & OR $(95 \% C I)$ \\
\hline CRP & $0.91(0.71-1.16)$ & $0.93(0.74-1.17)$ & $0.99(0.94-1.03)$ & $0.99(0.96-1.01)$ & $1.00(0.94-1.07)$ & $0.87(0.58-1.29)$ \\
\hline TNF- $\alpha$ & $0.80(0.63-1.02)^{\#}$ & $0.94(0.74-1.18)$ & $0.98(0.93-1.03)$ & $0.99(0.97-1.02)$ & $0.99(0.92-1.06)$ & $0.79(0.53-1.18)$ \\
\hline IL- $1 \beta$ & $1.13(0.88-1.45)$ & $0.94(0.74-1.18)$ & $1.05(1.00-1.11)^{\#}$ & $1.00(0.97-1.03)$ & $0.98(0.92-1.05)$ & $1.10(0.73-1.65)$ \\
\hline IFN- $\gamma$ & $1.13(0.89-1.45)$ & $1.13(0.89-1.45)$ & $1.06(1.01-1.12)^{*}$ & $1.04(1.01-1.07)^{* *}$ & $1.11(1.03-1.19)^{* *}$ & $1.54(1.01-2.34)^{*}$ \\
\hline IL-6 & $0.90(0.71-1.15)$ & $0.91(0.73-1.15)$ & $1.01(0.96-1.06)$ & $1.00(0.98-1.03)$ & $0.99(0.93-1.06)$ & $0.91(0.61-1.36)$ \\
\hline IL-12(p70) & $1.08(0.84-1.39)$ & $1.06(0.84-1.35)$ & $1.00(0.95-1.05)$ & $1.01(0.99-1.04)$ & 1.05 (0.98 - 1.12) & $1.19(0.78-1.81)$ \\
\hline \multicolumn{7}{|l|}{ Anti-inflammatory } \\
\hline IL-1RA & $1.10(0.86-1.42)$ & $1.17(0.92-1.49)$ & $1.07(1.02-1.13)^{* *}$ & $1.02(0.99-1.05)$ & $1.02(0.96-1.09)$ & $1.42(0.93-2.16)$ \\
\hline IL-4 & $0.96(0.75-1.22)$ & $0.91(0.72-1.14)$ & $1.03(0.98-1.08)$ & $1.01(0.98-1.04)$ & $0.99(0.93-1.06)$ & $0.96(0.65-1.44)$ \\
\hline IL-10 & $0.91(0.72-1.16)$ & $0.88(0.70-1.10)$ & $1.01(0.96-1.06)$ & $1.00(0.97-1.02)$ & $0.98(0.92-1.04)$ & $0.88(0.59-1.30)$ \\
\hline \multicolumn{7}{|c|}{ Cytokine ratios (pro-/anti-inflammatory) } \\
\hline TNF- $\alpha / \mathrm{IL}-4$ & $0.94(0.74-1.20)$ & $1.19(0.93-1.51)$ & $0.98(0.93-1.03)$ & $1.00(0.98-1.03)$ & $1.01(0.94-1.08)$ & $1.05(0.70-1.57)$ \\
\hline TNF- $\alpha / \mathrm{IL}-10$ & $0.75(0.58-0.97)^{*}$ & $1.16(0.91-1.47)$ & $0.97(0.92-1.02)$ & $0.99(0.97-1.02)$ & $0.99(0.92-1.05)$ & $0.84(0.56-1.26)$ \\
\hline IL- $1 \beta / \mathrm{IL}-1 \mathrm{RA}$ & $1.26(0.98-1.64)^{\#}$ & $1.04(0.83-1.32)$ & $0.99(0.94-1.04)$ & $1.00(0.98-1.03)$ & $1.00(0.94-1.07)$ & $1.16(0.77-1.74)$ \\
\hline
\end{tabular}




\begin{tabular}{|c|c|c|c|c|c|c|}
\hline \multicolumn{7}{|l|}{ Continued } \\
\hline IL-1 $\beta / \mathrm{IL}-10$ & $0.91(0.71-1.16)$ & $1.14(0.90-1.45)$ & $1.00(0.96-1.05)$ & $1.00(0.97-1.03)$ & $1.01(0.95-1.08)$ & $1.05(0.70-1.56)$ \\
\hline IL-1 $\beta / \mathrm{IL}-4$ & $0.98(0.77-1.24)$ & $1.13(0.89-1.42)$ & $0.97(0.92-1.02)$ & $0.99(0.96-1.02)$ & $0.98(0.92-1.05)$ & $0.96(0.65-1.43)$ \\
\hline IL-6/IL-10 & $0.92(0.72-1.17)$ & $1.16(0.92-1.47)$ & $1.00(0.95-1.05)$ & $1.01(0.98-1.03)$ & 1.02 (0.95 - 1.09) & $1.08(0.73-1.61)$ \\
\hline IL-6/IL-4 & $1.11(0.87-1.41)$ & $1.30(1.02-1.67)^{*}$ & $1.00(0.95-1.05)$ & $1.01(0.99-1.04)$ & $1.01(0.95-1.08)$ & $1.30(0.86-1.95)$ \\
\hline IFN- $\gamma / \mathrm{IL}-4$ & $1.14(0.89-1.46)$ & $1.23(0.96-1.56)$ & $1.01(0.96-1.06)$ & $1.02(0.99-1.05)$ & $1.05(0.98-1.12)$ & $1.37(0.91-2.07)$ \\
\hline IFN- $\gamma /$ IL10 & $1.09(0.85-1.39)$ & $1.17(0.92-1.48)$ & $1.05(1.00-1.11)^{\#}$ & $1.02(1.00-1.05)$ & $1.11(1.03-1.19)^{* *}$ & $1.47(0.97-2.24)^{\#}$ \\
\hline IL-12/IL-10 & $0.92(0.73-1.17)$ & $0.99(0.78-1.24)$ & $1.02(0.97-1.07)$ & $1.01(0.98-1.04)$ & $1.06(0.99-1.14)^{\#}$ & 1.07 (0.72 - 1.59) \\
\hline IL-12/IL-4 & $1.09(0.85-1.38)$ & $1.26(0.99-1.61)^{\#}$ & $1.00(0.95-1.05)$ & $1.02(0.99-1.04)$ & $1.04(0.97-1.11)$ & $1.30(0.87-1.95)$ \\
\hline \multicolumn{7}{|l|}{ Stress hormones } \\
\hline CORT & $1.13(0.88-1.45)$ & $0.95(0.75-1.21)$ & $0.99(0.95-1.04)$ & $1.02(0.99-1.04)$ & $1.00(0.93$ - 1.06) & $1.05(0.70-1.57)$ \\
\hline EPI & $0.83(0.64-1.07)$ & $0.84(0.66-1.08)$ & $0.98(0.93-1.03)$ & $1.00(0.97-1.03)$ & $0.99(0.92-1.06)$ & $0.75(0.49-1.15)$ \\
\hline NE & $0.87(0.68-1.11)$ & $0.91(0.72-1.17)$ & $0.94(0.89-0.99)^{*}$ & $0.99(0.96-1.02)$ & $0.96(0.90-1.03)$ & $0.75(0.49-1.14)$ \\
\hline
\end{tabular}

Controlling for demographics (age, education, gender, race), physical function (Duke Activity Status Index), depressive symptoms (BDI), OR = odds ratio, $\mathrm{CI}=$ confidence interval (biomarkers dichotomized: high $=$ top $1 / 3$ and low = bottom $2 / 3$ ), ${ }^{*} 0.05<\mathrm{p}<0.10,{ }^{*} \mathrm{p} \leq 0.05,{ }^{* *} \mathrm{p} \leq 0.01, \mathrm{n}=122-129$.

in the analyses. The average age of participants was 51.6 years (range 24 - 84), average education level was 15.1 years (range 4 - 31), and the majority were female (68.9\%) and white (53.0\%) (Table 1). The mean BDI score was $25.3 \pm 8.5$, confirming that our participants were moderately depressed. Cytokine profiling in serum samples revealed detectable levels of the acute phase protein, CRP, pro-inflammatory cytokines (TNF- $\alpha$, IL-1 $\beta$, IFN- $\gamma$, IL-6, and IL-12), and anti-inflammatory cytokines (IL-4, IL-1RA, IL-10). Pro-inflammatory-to-anti-inflammatory ratios were highest for IFN- $\gamma / \mathrm{IL}-10$ (1.3), TNF- $\alpha / \mathrm{IL}-10$ (1.1) and IFN- $\gamma / \mathrm{IL}-4(0.8)$, and lowest for IL-1 $\beta /$ IL-1RA (0.1), IL-1 $\beta / I L-4$ (0.1), IL-1 $\beta / I L-10$ (0.2), and IL-12/IL-4 (0.2).

Comparison to MIDUS. When MIDUS participants were compared to the present sample, the former were older (59.2 vs. 51.6 years, $\mathrm{p}<0.05)$, less likely to be female $(53.7 \%$ vs. $68.9 \%, \mathrm{p}<0.05)$, more likely to be white $(92.0 \%$ vs. $53.0 \%, \mathrm{p}<0.05)$, but had similar education ( 14.8 vs. $15.1, \mathrm{p}=0.34)$ (Table 1$)$. The average CES-D score (9.0, SD 10.3) was considerably below the usual cut-off of 16 or higher for significant depression, indicating that participants in the MIDUS study were largely not depressed. We did not statistically compare biomarkers due to their non-normal distributions. Instead, we provide the median value and range for the two pro-inflammatory markers (CRP, IL-6) and three stress hormones (cortisol, epinephrine, norepinephrine) common to both samples. Although levels of IL-6 were similar between samples, the median CRP in our participants with major depressive disorder was nearly three times that of MIDUS participants (4.4 vs. $1.3 \mathrm{ng} / \mathrm{ml}$ ). Likewise, median urinary cortisol level was three times higher (30.2 vs. $11.0 \mathrm{mg} / \mathrm{L}$ creatinine) in our depressed sample compared to MIDUS participants, urinary epinephrine was almost three times higher (5.4 vs. $1.8 \mathrm{mg} / \mathrm{L}$ ), and median urine norepinephrine level was about $50 \%$ higher (39.5 vs. $26.0 \mathrm{mg} / \mathrm{L}$ ). Given known relationships between depressive disorder, inflammatory markers, and stress hormones, these findings are expected and support the accurate determination of biomarker levels reported in the present study.

Biomarkers and depressive symptoms. In the present sample, we hypothesized that greater depression severity would be related to higher levels of pro-inflammatory biomarkers, lower anti-inflammatory cytokines, higher pro-/anti-inflammatory cytokine ratios, and higher urinary stress hormones. Table 2 describes the average level of depressive symptoms across low and high levels of biomarkers, where high represents the top one-third of values. Table 3 illustrates these relationships in multivariate analyses controlling for demographics and physical functioning. Neither bivariate nor multivariate analyses supported our hypothesis. If anything, the opposite was true. In bivariate analyses, severity of depressive symptoms tended to be lower among those with high levels of the pro-inflammatory cytokines TNF- $\alpha(22.9 \mathrm{vs.} 26.5 \mathrm{pg} / \mathrm{ml}, \mathrm{t}=-2.29, \mathrm{p}=0.02)$ and IL-1 $\beta(23.4 \mathrm{vs} .26 .3 \mathrm{pg} / \mathrm{ml}$, $\mathrm{t}=-1.78, \mathrm{p}=0.08)$ and in those with high levels of urinary cortisol (23.3 vs. $26.2 \mathrm{mg} / \mathrm{L}$ creatinine, $\mathrm{t}=-1.84, \mathrm{p}$ $=0.07$ ). Multivariate models controlling for demographics and physical functioning (Table 3 ) largely confirmed these findings.

Biomarkers and religious characteristics. We hypothesized that religious activities and attitudes would be 
lower among those with 1) high levels of pro-inflammatory markers, 2) low anti-inflammatory cytokines, 3) high pro-/anti-inflammatory cytokine ratios, and 4) high stress hormones. Table 2 presents the average level of religious involvement across low and high levels of biomarkers, and Table 4 shows these relationships controlled for demographics, depressive symptoms, and physical functioning. Again, neither bivariate nor multivariate analyses supported our hypotheses (including our specific hypothesis), and most associations were nonsignificant. While there were some significant and near-significant values, no discernible pattern in one direction or the other was identified (see Figures 1-3).

Bivariate analyses. For religious attendance, while frequency of attendance as expected tended to be lower in those with high levels of TNF- $\alpha$ ( $3.5 \mathrm{vs} .4 .0 \mathrm{pg} / \mathrm{ml}, \mathrm{t}=-1.75, \mathrm{p}=0.08)$ and high levels of the pro-/anti-inflammatory ratio TNF- $\alpha$ to IL-10 ( $3.4 \mathrm{vs} .4 .1 \mathrm{pg} / \mathrm{ml}, \mathrm{t}=-2.14, \mathrm{p}=0.03$ ), attendance tended to be higher in those with high levels of the pro-/anti-inflammatory ratio IL-1 $\beta$ to IL-1RA ( 4.2 vs. $3.7, t=1.79, \mathrm{p}=0.08$ ). Likewise, private religious activities such as prayer and scripture reading were more frequent among those with high levels of the pro-/anti-inflammatory ratio IL-6 to IL-4 ( 4.1 vs. $3.4, t=2.31, \mathrm{p}=0.02)$, high levels of the pro-/anti-inflammatory ratio IFN- $\gamma$ to IL-4 ( 4.0 vs. $3.4, t=2.0, p=0.04$ ), and high levels of the pro-/anti-inflammatory ratio

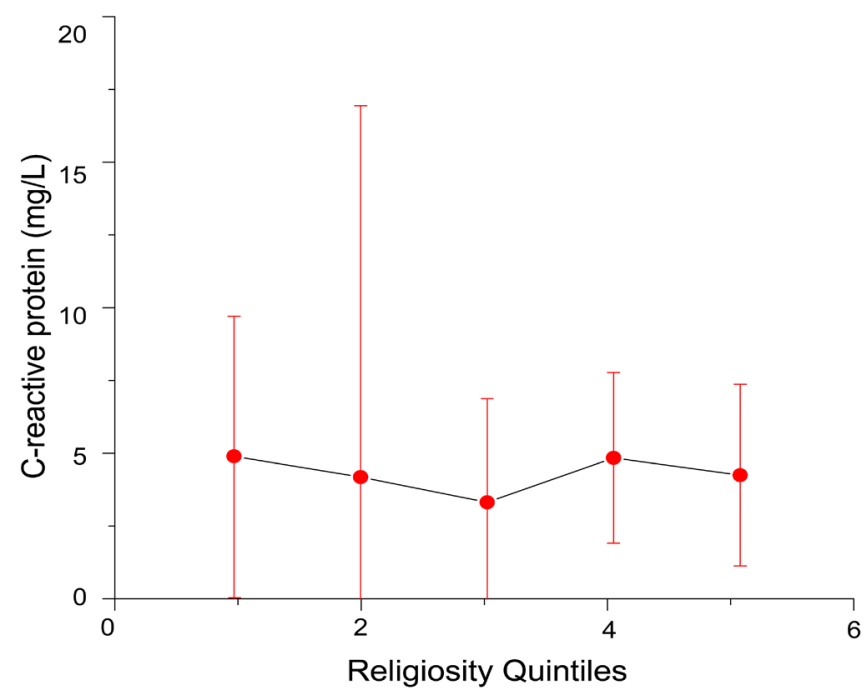

Figure 1. C-reactive protein (median, interquartile range) by overall religiosity $(\rho=0.0004, \mathrm{p}=\mathrm{ns}, \mathrm{n}=129)$.

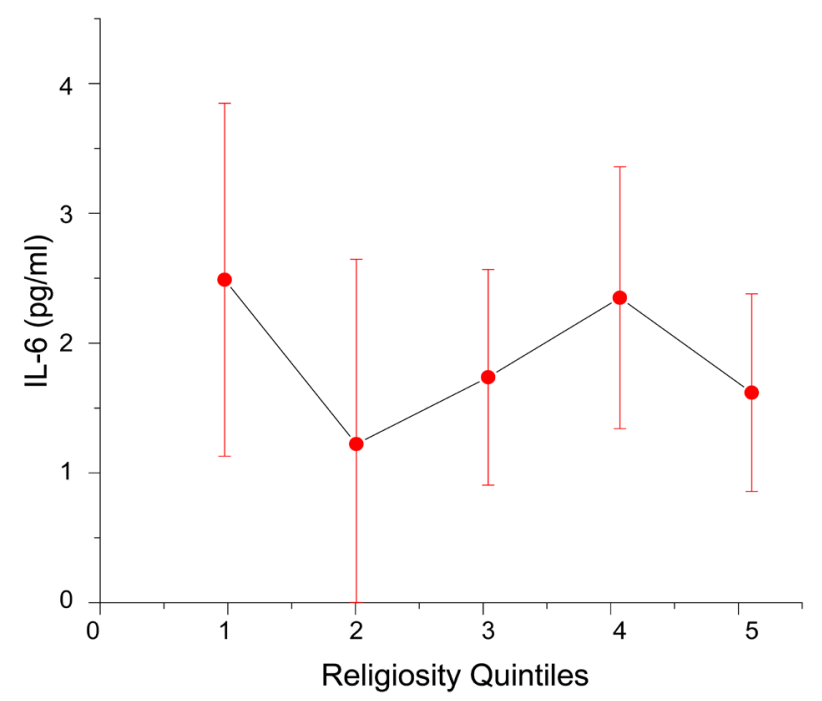

Figure 2. Interleukin-6 (median, interquartile range) by overall religiosity $(\rho=-0.015, \mathrm{p}=\mathrm{ns}, \mathrm{n}=126)$. 


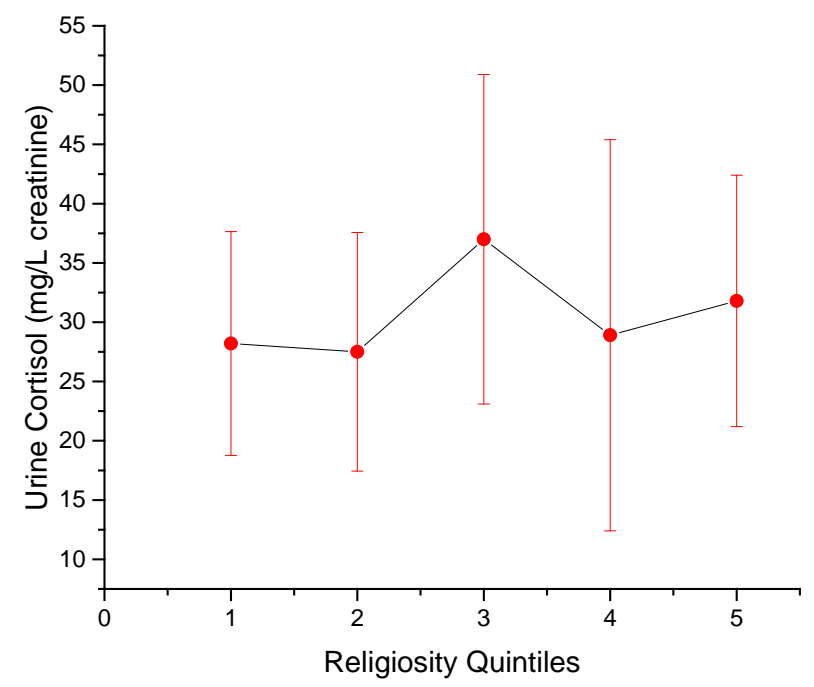

Figure 3. Urine cortisol (median, interquartile range) by overall religiosity $(\rho=0.054, \mathrm{p}=\mathrm{ns}, \mathrm{n}=127)$.

IL-12 to IL4 (4.1 vs. 3.4, $\mathrm{t}=2.1, \mathrm{p}=0.04)$. Similarly, intrinsic religiosity tended to be higher in those with high levels of the pro-inflammatory marker IFN- $\gamma(36.7 \mathrm{vs} .34 .1, \mathrm{t}=1.68, \mathrm{p}=0.09)$ and high levels of the pro-/antiinflammatory ratio IFN- $\gamma$ to IL-10 (37.1 vs. $34.0, t=2.07, p=0.04)$. One of the few findings consistent with our hypothesis, intrinsic religiosity was higher in those with high levels of the anti-inflammatory cytokine IL-1RA (37.5 vs. 33.5, $t=2.6, p=0.01$ ). Inconsistent with our hypothesis, however, daily religious and spiritual experiences tended to be more common among those with high levels of the pro-inflammatory IFN- $\gamma$ (61.6 vs. 55.7, $\mathrm{t}$ $=1.97, \mathrm{p}=0.05)$ and high levels of the pro-/anti-inflammatory ratio IFN- $\gamma$ to IL-10 (61.5 vs. 55.9, $\mathrm{t}=1.85, \mathrm{p}=$ 0.07). Religious coping was also higher among those with high levels of the pro-inflammatory IFN- $\gamma$ (31.3 vs. 28.5, $\mathrm{t}=2.50, \mathrm{p}=0.01$ ), high levels of the pro-/anti-inflammatory ratio IFN- $\gamma$ to IL-10 (31.9 vs. 28.3, $\mathrm{t}=3.15, \mathrm{p}$ $=0.002)$, and high levels of the pro-/anti-inflammatory ratio IL-12 to IL-10 (31.0 vs. $28.7, \mathrm{t}=1.91, \mathrm{p}=0.06)$. No significant associations were found with overall religiosity, although religiosity tended to be higher in those with high levels of IFN- $\gamma$ (pro-inflammatory), IL-1RA (anti-inflammatory), and IFN- $\gamma$ to IL-10 ratio (pro-inflammatory).

Multivariate analyses. Multivariate analyses (Table 4) essentially confirmed bivariate analyses, with the vast majority of associations non-significant and a mixed pattern of expected and unexpected findings. Religious attendance tended to be lower in those with high levels of the pro-inflammatory TNF- $\alpha$ (OR $=0.80,95 \% \mathrm{CI}=$ 0.63 - 1.02) and high TNF- $\alpha$ to IL-10 ratio (OR $=0.75,95 \% \mathrm{CI}=0.58-0.97)$, but was higher in those with high pro-/anti-inflammatory ratio IL- $1 \beta$ to IL-1RA $(\mathrm{OR}=1.26,95 \% \mathrm{CI}=0.98-1.64)$. Private prayer and scripture reading were also higher in those with high pro-/anti-inflammatory ratio IL-6 to IL-4 (OR $=1.30,95 \%$ CI $=1.02$ - 1.67) and high pro-/anti-inflammatory ratio IL-12 to IL-4 (OR = 1.26, 95\% CI = 0.99 - 1.61). Likewise, intrinsic religiosity was higher among those with high levels of pro-inflammatory cytokines IL-1 $\beta$ (OR $=1.05$, 95\% $\mathrm{CI}=1.00-1.11), \mathrm{IFN}-\gamma(\mathrm{OR}=1.06,95 \% \mathrm{CI}=1.01-1.12)$, and high pro-/anti-inflammatory ratio IFN- $\gamma$ to IL-10 (OR $=1.05,95 \%$ CI = $1.00-1.11)$, all contrary to our hypothesis. As expected, though, intrinsic religiosity was higher among those with high levels of the anti-inflammatory cytokine IL-1RA $(\mathrm{OR}=1.07,95 \% \mathrm{CI}=$ $1.02-1.13)$ and was lower in those with high levels of urinary norepinephrine (OR $=0.94,95 \% \mathrm{CI}=0.89$ 0.99). But, contrary to expectations, daily spiritual experiences were significantly higher among those with high levels of IFN- $\gamma(\mathrm{OR}=1.04,95 \% \mathrm{CI}=1.01-1.07)$, and religious coping was more common among those with high levels of IFN- $\gamma(\mathrm{OR}=1.11,95 \% \mathrm{CI}=1.03-1.19)$, high levels of IFN- $\gamma$ to IL-10 ratio (OR $=1.11,95 \%$ CI $=1.03-1.19)$, and high levels of IL-12 to IL-10 ratio $(\mathrm{OR}=1.06,95 \% \mathrm{CI}=0.99-1.14)$. Similarly, overall religiosity tended to be higher among those with high levels of IFN- $\gamma(\mathrm{OR}=1.54,95 \% \mathrm{CI}=1.01-2.34)$ and high levels of the IFN- $\gamma$ to IL-10 ratio (OR $=1.47,95 \% \mathrm{CI}=0.97-2.24)$. Thus, if any consistent pattern was found in these relationships, religious activities and attitudes were related to higher levels of pro-inflammatory markers, the exact opposite of our hypothesis. 


\section{Discussion}

This is one of the first studies to provide a detailed examination of associations between religious practices/experiences and a wide range of stress biomarkers in persons with major depressive disorder and chronic medical illness, while controlling for demographics, depressive symptoms and physical functioning. As expected, inflammatory markers and stress hormones were higher in the present sample than in a community sample of relatively healthy middle-aged adults. Contrary to expectations, depressive symptoms were largely unrelated to stress biomarkers in the current sample, and when related, were actually lower among those with higher levels of pro-inflammatory cytokines (TNF- $\alpha, \mathrm{IL}-1 \beta$ ). This contrasts with what is reported in most of the literature, where those with more severe depression are usually found to have higher levels of pro-inflammatory cytokines, especially IL-6, TNF- $\alpha$ and IFN- $\gamma$ [67] [68]. All participants in the current study, however, had major depressive disorder to start with and the range of depressive symptoms was limited due to its mild to moderate severity (BDI 10 to 42). The presence of co-morbid chronic medical illness may also have added to the variability seen in biomarkers found here.

Likewise, religious practices and experiences (RPE) were largely unrelated to these stress biomarkers, and there was no evidence in favor of our primary hypothesis that overall religiosity would be related to lower levels of CRP, IL-6, or urinary cortisol (Figures 1-3). In fact, no clearly discernible pattern of association was found between RPE and inflammatory cytokine or stress hormone markers among the 138 analyses done here. Consistent with our hypothesis, RPE were higher among those with high levels of the anti-inflammatory cytokine IL-1RA and lower in those with high levels of urinary norepinephrine. However, RPE were also higher in those with high pro-inflammatory cytokines, especially IFN- $\gamma$, IFN- $\gamma /$ IL-4, and IFN- $\gamma /$ IL-10, which is contrary to our hypothesis. To our knowledge, this is the first study to report a link between religious activity and an anti-inflammatory cytokine (IL-1RA). Others, however, have reported an increase in IFN- $\gamma$ with spiritual practices [69]-[71], which may result from the complex relationship between IFN- $\gamma$, depressive symptoms, and stress. On the one hand, IFN- $\gamma$ increases in major depression [72] [73] and decreases in response to treatment [74] [75], at least among studies in men. On the other hand, IFN- $\gamma$ is suppressed by cortisol, decreases during acute psychological stress [24], and may increase in response to treatment, especially in depressed women who may have lower IFN- $\gamma$ levels than depressed men [74] [76].

This is also to our knowledge the first report of a relationship between greater intrinsic religiosity and lower urinary norepinephrine, a stress hormone known to increase in depression [77] and be associated with a worse prognosis in cardiovascular diseases [78]. In a 32-year prospective study that compared 144 Catholic sisters with 138 healthy laywomen from a community in Italy, investigators reported that urinary norepinephrine was significantly lower in sisters at every six-year evaluation throughout the study period [79]. Furthermore, at least two studies have found that spiritual interventions (meditation) lowered serum or urinary norepinephrine in healthy adults [80] and in those with congestive heart failure [81].

\section{Limitations}

Numerous limitations exist that should be taken into account when generalizing or interpreting the findings reported here. First, this was a relatively small sample and a sample of convenience (volunteers), making it difficult to generalize results to other populations. Second, the sample was made up of people with chronic medical illnesses taking an assortment of medications that could influence levels of inflammatory markers and stress hormones and make it difficult to detect more subtle associations with either depressive symptoms or RPE. Third, all participants in this study had major depressive disorder, and most median biomarker levels were three times that greater than in the comparison community population, thus ceiling effects may have been an issue. Fourth, as noted above, given the multiple statistical comparisons made here, at least 1 in 20 comparisons would be expected to be significant based on chance alone, increasing the likelihood that significant findings reported here were due to Type I error. Nevertheless, the study also has several strengths. Participants were drawn from two different sites, the East coast and the West coast, increasing the likelihood of generalizability; analyses were carefully controlled for demographics and physical health; and this is the first study to examine such associations in persons with comorbid major depressive disorder and chronic medical illness. Furthermore, the breadth of religious characteristics assessed in this study is unparalleled in the literature for studies examining relationships between religiosity and biomarkers [82]. 


\section{Conclusion}

Although the median and range inflammatory markers and stress hormones measured here were higher than those from a healthy non-depressed community sample as expected, we found little evidence for a relationship between depressive symptoms and these biomarkers in persons with major depressive disorder and chronic illness. Furthermore, we found no consistent pattern between religious practices or experiences and either inflammatory markers or stress hormones in these cross-sectional analyses (with the exception of higher levels of the anti-inflammatory cytokine IL-1RA, higher pro-inflammatory cytokine IFN- $\gamma$, and lower urinary norepinephrine). Future research, particularly the ongoing clinical trial that sample is the baseline for, is needed to help determine whether religious interventions for major depression among those with chronic medical illness can help to reverse the high levels of pro-inflammatory markers and stress hormone levels associated with depression.

\section{Funding}

Funding support provided by the John Templeton Foundation.

\section{References}

[1] Kitchell, M.A., Barnes, R.F., Veith, R.C., et al. (1982) Screening for Depression in Hospitalized Geriatric Medical Patients. Journal of the American Geriatrics Society, 30, 174-177.

[2] Koenig, H.G., George, L.K., Peterson, B.L. and Pieper, C.F. (1997) Depression in Medically Ill Hospitalized Older Adults: Prevalence, Correlates, and Course of Symptoms Based on Six Diagnostic Schemes. American Journal of Psychiatry, 154, 1376-1383.

[3] Rosemann, T., Backenstrass, M., Joest, K., Rosemann, A., Szecsenyi, J. and Laux, G. (2007) Predictors of Depression in a Sample of 1021 Primary Care Patients with Osteoarthritis. Arthritis \& Rheumatism, 57, 415-422. http://dx.doi.org/10.1002/art.22624

[4] Turner, R.J. and Noh, S. (1988) Physical Disability and Depression: A Longitudinal Analysis. Journal of Health and Social Behavior, 29, 23-37. http://dx.doi.org/10.2307/2137178

[5] Penninx, B.W.J.H., Leveille, S., Ferrucci, L., van Eijk, J. and Guralnik, J.M. (1999) Exploring the Effect of Depression on Physical Disability: Longitudinal Evidence from the Established Populations for Epidemiologic Studies of the Elderly. American Journal of Public Health, 89, 1346-1352. http://dx.doi.org/10.2105/AJPH.89.9.1346

[6] Meltzer, H., Begginton, P., Brugha, T., McManus, S., Raj, D., Dennis, M.S. and Jenkins, R. (2012) Physical Ill Health, Disability, Dependence and Depression: Results from the 2007 National Survey of Psychiatric Morbidity among Adults in England. Disability and Health Journal, 5, 102-110. http://dx.doi.org/10.1016/j.dhjo.2012.02.001

[7] Pew Forum (2007) U.S. Religious Landscape Survey. http://religions.pewforum.org/

[8] The Gallup Poll (2009) State of the States: Importance of Religion. http://www.gallup.com/poll/114022/state-states-importance-religion.aspx

[9] Koenig, H.G. (1998) Religious Beliefs and Practices of Hospitalized Medically Ill Older Adults. International Journal of Geriatric Psychiatry, 13, 213-224. http://dx.doi.org/10.1002/(SICI)1099-1166(199804)13:4<213::AID-GPS755>3.0.CO;2-5

[10] Evans, D.L., Ten, T.R., Douglas, S.D., et al. (2002) Association of Depression with Viral Load, CD8 T Lymphocytes, and Natural Killer Cells in Women with HIV Infection. American Journal of Psychiatry, 159, 1752-1759. http://dx.doi.org/10.1176/appi.ajp.159.10.1752

[11] Zautra, A.J., Yocum, D.C., Villanueva, I., et al. (2004) Immune Activation and Depression in Women with Rheumatoid Arthritis. Journal of Rheumatology, 31, 457-463.

[12] Thaker, P.H., Lutgendorf, S.K. and Sood, A.K. (2007) The Neuroendocrine Impact of Chronic Stress on Cancer. Cell Cycle, 6, 430-433. http://dx.doi.org/10.4161/cc.6.4.3829

[13] Lutgendorf, S.K., Lamkin, D.M., DeGeest, K., Anderson, B., Dao, M., McGinn, S., et al. (2008) Depressed and Anxious Mood and T-Cell Cytokine Expressing Populations in Ovarian Cancer Patients. Brain, Behavior and Immunity, 22, 890-900. http://dx.doi.org/10.1016/j.bbi.2007.12.012

[14] Lutgendorf, S.K., DeGeest, K., Sung, C.Y., Arevalo, J.M., Penedo, F. and Lucci 3rd, J. (2009) Depression, Social Support and Beta-Adrenergic Transcription Control in Human Ovarian Cancer. Brain, Behavior and Immunity, 23, 176-183. http://dx.doi.org/10.1016/j.bbi.2008.04.155

[15] Irwin, M.R. and Miller, A.H. (2007) Depressive Disorders and Immunity: 20 Years of Progress and Discovery. Brain, Behavior and Immunity, 21, 374-383. http://dx.doi.org/10.1016/j.bbi.2007.01.010 
[16] Leonard, B.E. and Myint, A. (2009) The Psychoneuroimmunology of Depression. Human Psychopharmacology, 24, $165-175$.

[17] Rosenkranz, M.A., Jackson, D.C., Dalton, K.M., Dolski, I., Ryff, C.D., Singer, B.H., Muller, D., Kalin, N.H. and Davidson, R.J. (2003) Affective Style and in Vivo Immune Response: Neurobehavioral Mechanisms. Proceedings of the National Academy of Sciences, 100, 11148-11152. http://dx.doi.org/10.1073/pnas.1534743100

[18] Miller, A.H. (1998) Neuroendocrine and Immune System Interactions in Stress and Depression. Psychiatric Clinics of North America, 21, 443-463. http://dx.doi.org/10.1016/S0193-953X(05)70015-0

[19] Kiecolt-Glaser, J.K., Preacher, K.J., MacCallum, R.C., Atkinson, C., Malarkey, W.B. and Glaser, R. (2003) Chronic Stress and Age-Related Increases in the Proinflammatory Cytokine IL-6. Proceedings of the National Academy of Sciences, 100 , 9090-9095. http://dx.doi.org/10.1073/pnas.1531903100

[20] Howren, M.B., Lamkin, D.M. and Suls, J. (2009) Associations of Depression with C-Reactive Protein, Il-1 and Il-6: A Meta-Analysis. Psychosomatic Medicine, 71, 171-186. http://dx.doi.org/10.1097/PSY.0b013e3181907c1b

[21] Hestad, K.A., Tonseth, S., Stoen, C.D., Ueland, T. and Aukrust, P. (2003) Raised Plasma Levels of Tumor Necrosis Factor Alpha in Patients with Depression: Normalization during Electroconvulsive Therapy. Journal of Electroconvulsive Therapy, 19, 183-188.

[22] Guglu, C., Kara, S.H., Caliyurt, O., Vardar, E. and Abay, E. (2003) Increased Serum Tumor Necrosis Factor-Alpha Levels and Treatment Response in Major Depressive Disorder. Psychopharmacology, 170, 429-433. http://dx.doi.org/10.1007/s00213-003-1566-z

[23] Leonard, B.E. and Myint, A. (2009) The Psychoneuroimmunology of Depression. Human Psychopharmacology, 24, 165-175.

[24] Zorrilla, E.P., Luborsky, L., McKay, J.R., Rosenthal, R., Houldin, A., Tx, A., McCorkle, R., Seligman, D.A. and Schmidt, K. (2001) The Relationship of Depression and Tressors to Immunoloigcal Assays: A Meta-Analytic Review. Brain, Behavior and Immunity, 15, 199-226. http://dx.doi.org/10.1006/brbi.2000.0597

[25] Hestad, K.A., Tonseth, S., Stoen, C.D., Ueland, T. and Aukrust, P. (2003) Raised Plasma Levels of Tumor Necrosis Factor Alpha in Patients with Depression: Normalization during Electroconvulsive Therapy. Journal of Electroconvulsive Therapy, 19, 183-188.

[26] Guglu, C., Kara, S.H., Caliyurt, O., Vardar, E. and Abay, E. (2003) Increased Serum Tumor Necrosis Factor-Alpha Levels and Treatment Response in Major Depressive Disorder. Psychopharmacology, 170, 429-433. http://dx.doi.org/10.1007/s00213-003-1566-z

[27] Hannestad, J., DellaGiola, N. and Bloch, M. (2011) The Effect of Antidepressant Medication Treatment on Serum Levels of Inflammatory Cytokines: A Meta-Analysis. Neuropsychopharmacology, 36, 2452-2459. http://dx.doi.org/10.1038/npp.2011.132

[28] Castanon, N., Leonard, B.E., Neveu, P.J. and Yirmiya, R. (2002) Effects of Antidepressants on Cytokine Production and Actions. Brain, Behavior and Immunity, 16, 569-574. http://dx.doi.org/10.1016/S0889-1591(02)00008-9

[29] Dahl, J., Ormstad, H., Aas, H.C.D., Malt, U.F., Bendtz, L.T., Sandvik, L., Brundin, L. and Andreassen, O.A. (2014) The Plasma Level of Various Cytokines Are Increased during Ongoing Depression and Are Reduced to Normal Levels after Recovery. Psychoneuroendocrinology, 45, 77-86. http://dx.doi.org/10.1016/j.psyneuen.2014.03.019

[30] van Middendorp, H., Geenen, R., Sorbi, M.J., van Doornen, L.J. and Bijlsma, J.W. (2009) Health and Physiological Effects of an Emotional Disclosure Intervention Adapted for Application at Home: A Randomized Clinical Trial in Rheumatoid Arthritis. Psychotherapy \& Psychosomatics, 78, 145-151. http://dx.doi.org/10.1159/000206868

[31] Berk, L.S., Tan, S. and Tan, L.G. (2009) Mirthful Laughter, as Adjunct Therapy in Diabetic Care, Increases HDL Cholesterol and Attenuates Inflammatory Cytokines and CRP and Possible CVD Risk. Proceedings of the 2009 American Physiological Society Section (FASEB), New Orleans, 18-22 April 2009, Abstract No. 370.

[32] Antoni, M.H., Lechner, S., Diaz, A., Vargas, S., Holley, H., Phillips, K., McGregor, B., Carver, C.S. and Blomberg, B. (2009) Cognitive Behavioral Stress Management Effects on Psychosocial and Physiological Adaptation in Women Undergoing Treatment for Breast Cancer. Brain, Behavior and Immunity, 23, 580-591. http://dx.doi.org/10.1016/j.bbi.2008.09.005

[33] Roberts, A.D.L., Papadopoulos, A.S., Wessely, S., Chalder, T. and Cleare, A.J. (2009) Salivary Cortisol Output before and after Cognitive Behavioral Therapy for Chronic Fatigue Syndrome. Journal of Affective Disorders, 115, 280-286. http://dx.doi.org/10.1016/j.jad.2008.09.013

[34] Koenig, H.G., Cohen, H.J., Blazer, D.G., Pieper, C., Meador, K.G., Shelp, F., Goli, V. and Di Pasquale, R. (1992) Religious Coping and Depression in Elderly Hospitalized Medically Ill Men. American Journal of Psychiatry, 149, 16931700.

[35] Koenig, H.G. (2007) Religion and Remission of Depression in Medical Inpatients with Heart Failure/Pulmonary Disease. Journal of Nervous and Mental Disease, 195, 389-395. 
[36] Koenig, H.G., George, L.K. and Peterson, B.L. (1998) Religiosity and Remission of Depression in Medically Ill Older Patients. American Journal of Psychiatry, 155, 536-542.

[37] Koenig, H.G., Cohen, H.J., George, L.K., Hays, J.C., Larson, D.B. and Blazer, D.G. (1997) Attendance at Religious Services, Interleukin-6 and Other Biological Indicators of Immune Function in Older Adults. International Journal of Psychiatry in Medicine, 27, 233-250. http://dx.doi.org/10.2190/40NF-Q9Y2-0GG7-4WH6

[38] Lutgendorf, S.K., Russell, D., Ullrich, P., Harris, T.B. and Wallace, R. (2004) Religious Participation, Interleukin-6 and Mortality in Older Adults. Health Psychology, 23, 465-475. http://dx.doi.org/10.1037/0278-6133.23.5.465

[39] King, D.E., Mainous 3rd, A.G. and Pearson, W.S. (2002) C-Reactive Protein, Diabetes and Attendance at Religious Services. Diabetes Care, 25, 1172-1176. http://dx.doi.org/10.2337/diacare.25.7.1172

[40] Dedert, E.A., Studts, J.L., Weissbecker, I., Salmon, P.G., Banis, P.L. and Sephton, S.E. (2004) Private Religious Practice: Protection of Cortisol Rhythms among Women with Fibromyalgia. International Journal of Psychiatry in Medicine, 34, 61-77. http://dx.doi.org/10.2190/2Y72-6H80-BW93-U0T6

[41] Tartaro, J., Luecken, L.J. and Gunn, H.E. (2005) Exploring Heart and Soul: Effects of Religiosity/Spirituality and Gender on Blood Pressure and Cortisol Stress Responses. Journal of Health Psychology, 10, 753-766. http://dx.doi.org/10.1177/1359105305057311

[42] Ironson, G., Soloman, G.F., Balbin, E.G., O’Cleirigh, C., George, A., Kumar, M., et al. (2002) The Ironson-Woods Spirituality/Religiousness Index Is Associated with Long Survival, Health Behaviors, Less Distress and Low Cortisol in People with HIV/AIDS. Annals of Behavioral Medicine, 24, 34-48. http://dx.doi.org/10.1207/S15324796ABM2401_05

[43] Carrico, A.W., Ironson, G., Antoni, M.H., Lechner, S.C., Duran, R.E., Kumar, M., et al. (2006) A Path Model of the Effects of Spirituality on Depressive Symptoms and 24-h Urinary-Free Cortisol in HIV-Positive Persons. Journal of Psychosomatic Research, 61, 51-58. http://dx.doi.org/10.1016/j.jpsychores.2006.04.005

[44] Chan, C.L., Ho, R.T., Lee, P.W., Cheng, J.Y., Leung, P.P., Foo, W., et al. (2006) A Randomized Controlled Trial of Psychosocial Interventions Using the Psychophysiological Framework for Chinese Breast Cancer Patients. Journal of Psychosocial Oncology, 24, 3-26. http://dx.doi.org/10.1300/J077v24n01 02

[45] Pace, T.W., Negi, L.T., Adame, D.D., Cole, S.P., Sivilli, T.I., Brown, T.D., et al. (2009) Effect of Compassion Meditation on Neuroendocrine, Innate Immune and Behavioral Responses to Psychosocial Stress. Psychoneuroendocrinology, 34, 87-98. http://dx.doi.org/10.1016/j.psyneuen.2008.08.011

[46] Witek-Janusek, L., Albuquerque, K., Chroniak, K.R., Chroniak, C., Durazo-Arvizu, R. and Mathews, H.L. (2008) Effect of Mindfulness Based Stress Reduction on Immune Function, Quality of Life and Coping in Women Newly Diagnosed with Early Stage Breast Cancer. Brain, Behavior and Immunity, 22, 969-981. http://dx.doi.org/10.1016/j.bbi.2008.01.012

[47] Jevning, R., Wilson, A.F. and Davidson, J.M. (1978) Adrenocortical Activity during Meditation. Hormones \& Behavior, 10, 54-60. http://dx.doi.org/10.1016/0018-506X(78)90024-7

[48] Sudsuang, R., Chentanez, V. and Veluvan, K. (1991) Effect of Buddhist Meditation on Serum Cortisol and Total Protein Levels, Blood Pressure, Pulse Rate, Lung Volume and Reaction Time. Physiology \& Behavior, 50, 543-548. http://dx.doi.org/10.1016/0031-9384(91)90543-W

[49] Walton, K.G., Fields, J.Z., Levitsky, D.K., Harris, D.A., Pugh, N.D., Schneider, R.H., et al. (2004) Lowering Cortisol and CVD Risk in Postmenopausal Women: A Pilot Study Using the Transcendental Meditation Program. Annals of the New York Academy of Sciences, 1032, 211-215. http://dx.doi.org/10.1196/annals.1314.023

[50] Michalsen, A., Grossman, P., Acil, A., Langhorst, J., Ludtke, R., Esch, T., et al. (2005) Rapid Stress Reduction and Anxiolysis among Distressed Women as a Consequence of a Three-Month Intensive Yoga Program. Medical Science Monitor, 11, CR555-CR561.

[51] Witek-Janusek, L., Albuquerque, K., Chroniak, K.R., Chroniak, C., Durazo-Arvizu, R. and Mathews, H.L. (2008) Effect of Mindfulness Based Stress Reduction on Immune Function, Quality of Life and Coping in Women Newly Diagnosed with Early Stage Breast Cancer. Brain, Behavior and Immunity, 22, 969-981. http://dx.doi.org/10.1016/j.bbi.2008.01.012

[52] Jin, P. (1992) Efficacy of Tai Chi, Brisk Walking, Meditation and Reading in Reducing Mental and Emotional Stress. Journal of Psychosomatic Research, 36, 361-370. http://dx.doi.org/10.1016/0022-3999(92)90072-A

[53] Curiati, J.A., Bocchi, E., Freire, J.O., Arantes, A.C., Braga, M., Garcia, Y., et al. (2005) Meditation Reduces Sympathetic Activation and Improves the Quality of Life in Elderly Patients with Optimally Treated Heart Failure: A Prospective Randomized Study. Journal of Alternative and Complementary Medicine, 11, 465-472. http://dx.doi.org/10.1089/acm.2005.11.465

[54] Hlatky, M.A., Boineau, R.E., Higginbotham, M.B., Lee, K.L., Mark, D.B., Califf, R.M., Cobb, F.R. and Pryor, D.B. (1989) A Brief Self-Administered Questionnaire to Determine Functional Capacity (The Duke Activity Status Index). 
American Journal of Cardiology, 64, 651-654. http://dx.doi.org/10.1016/0002-9149(89)90496-7

[55] Koenig, H.G. (1996) An Abbreviated Mini-Mental State Exam for Medically Ill Elders. Journal of the American Geriatrics Society, 44, 215-216.

[56] Sheehan, B.V., Lecrubier, Y., Sheehan, K.H., et al. (1998) The Mini International Neuropsychiatric Interview (MINI): The Development and Validation of Structured Diagnostic Psychiatric Interview for DSM-IV and ICD-10. Journal of Clinical Psychiatry, 59, 22-33.

[57] Beck, A.T., Ward, C.H., Mendelson, M., Mock, J. and Erbaugh, J. (1961) An Inventory for Measuring Depression. Archives of General Psychiatry, 4, 561-571. http://dx.doi.org/10.1001/archpsyc.1961.01710120031004

[58] Koenig, H.G., Meador, K.G. and Parkerson, G. (1997) Religion Index for Psychiatric Research. American Journal of Psychiatry, 154, 885-886.

[59] Hoge, D.R. (1972) A Validated Intrinsic Religious Motivation Scale. Journal for the Scientific Study of Religion, 11, 369-376. http://dx.doi.org/10.2307/1384677

[60] Underwood, L.G. and Teresi, J.A. (2002) The Daily Spiritual Experiences Scale: Development, Theoretical Description, Reliability, Exploratory Factor Analysis and Preliminary Construct Validity Using Health-Related Data. Annals of Behavioral Medicine, 24, 22-33. http://dx.doi.org/10.1207/S15324796ABM2401_04

[61] Pargament, K.I., Smith, B.W., Koenig, H.G. and Perez, L. (1998) Patterns of Positive and Negative Religious Coping with Major Life Stressors. Journal for the Scientific Study of Religion, 37, 710-724. http://dx.doi.org/10.2307/1388152

[62] Midlife in the United States (2014) A National Longitudinal Study of Health and Well-Being. http://www.midus.wisc.edu/

[63] National Archive of Computerized Data on Aging (2014) National Survey of Midlife Development in the United States (MIDUS II): Biomarker Project, 2004-2009 (ICPSR 29282). https://www.icpsr.umich.edu/icpsrweb/NACDA/studies/29282?sortBy=5

[64] Love, G.D., Seeman, T.E., Weinstein, M. and Ryff, C.D. (2010) Bioindicators in the MIDUS National Study: Protocol, Measures, Sample and Comparative Context. Journal of Aging and Health, 22, 1059-1080. http://dx.doi.org/10.1177/0898264310374355

[65] Ryff, C.D., Seeman, C.D. and Weinstein, M. (2010) National Survey of Midlife Development in the United States (MIDUS II): Biomarker Project, 2004-2009, Blood, Urine and Saliva Data Documentation. University of Wisconsin, Institute on Aging, Madison.

[66] Aziz, N., Fahey, J.L., Detels, R. and Butch, A.W. (2003) Analytical Performance of a Highly Sensitive C-Reactive Protein-Based Immunoassay and the Effects of Laboratory Variables on Levels of Protein in Blood. Clinical and Diagnostic Laboratory Immunology, 10, 652-657.

[67] Kiecolt-Glaser, J.K., Preacher, K.J., MacCallum, R.C., Atkinson, C., Malarkey, W.B. and Glaser, R. (2003) Chronic Stress and Age-Related Increases in the Proinflammatory Cytokine IL-6. Proceedings of the National Academy of Sciences, 100, 9090-9095. http://dx.doi.org/10.1073/pnas.1531903100

[68] Dowlati, Y., Herrmann, N., Swardfager, W., Liu, H., Sham, L., Reim, E.K. and Lanctot, K.L. (2010) A Meta-Analysis of Cytokines in Major Depression. Biological Psychiatry, 67, 446-457. http://dx.doi.org/10.1016/j.biopsych.2009.09.033

[69] Jones, B.M. (2001) Changes in Cytokine Production in Healthy Subjects Practicing Guolin Qigong: A Pilot Study. BMC Complementary and Alternative Medicine, 1, 8. http://dx.doi.org/10.1186/1472-6882-1-8

[70] McCain, N.L., Gray, D.P., Elswick, R.K., Robins, J.W., Tuck, I., Walter, J.M., et al. (2008) A Randomized Clinical Trial of Alternative Stress Management Interventions in Persons with HIV Infection. Journal of Consulting and Clinical Psychology, 76, 431-441. http://dx.doi.org/10.1037/0022-006X.76.3.431

[71] Witek-Janusek, L., Albuquerque, K., Chroniak, K.R., Chroniak, C., Durazo-Arvizu, R. and Mathews, H.L. (2008) Effect of Mindfulness Based Stress Reduction on Immune Function, Quality of Life and Coping in Women Newly Diagnosed with Early Stage Breast Cancer. Brain, Behavior and Immunity, 22, 969-981. http://dx.doi.org/10.1016/j.bbi.2008.01.012

[72] Simon, N.M., McNamara, K., Chow, C.W., Maser, R.S., Papakostas, G.I. and Pollack, M.H. (2008) A Detailed Examination of Cytokine Abnormalities in Major Depressive Disorder. European Neuropsychopharmacology, 18, 230233. http://dx.doi.org/10.1016/j.euroneuro.2007.06.004

[73] Gabbay, V., Klein, R.G., Alonso, C.M., Babb, J.S., Nishawal, M., De Jesus, G., Hirsch, G.S., Hottinger-Blanc, P. and Gonzalez, C.J. (2009) Immune System Dysregulation in Adolescent Major Depressive Disorder. Journal of Affective Disorders, 115, 177-182. http://dx.doi.org/10.1016/j.jad.2008.07.022

[74] Myint, A.M., Leonard, B.E., Stenbusch, H.W.M. and Kim, Y.K. (2005) Th1, Th2 and Th3 Cytokine Alterations in Major Depression. Journal of Affective Disorders, 88, 167-173. http://dx.doi.org/10.1016/j.jad.2005.07.008 
[75] Tsao, C.W., Lin, Y.S., Chen, C.C., Bai, C.H. and Wu, S.R. (2006) Cytokines and Serotonin Transporter in Patients with Major Depression. Progress in Neuro-Psychopharmacology and Biological Psychiatry, 30, 899-905. http://dx.doi.org/10.1016/j.pnpbp.2006.01.029

[76] Kim, Y.K., Na, K.S., Shin, K.H., Jung, H.Y., Choi, S.H. and Kim, J.B. (2007) Cytokine Imbalance in the Pathophysiology of Major Depressive Disorder. Progress in Neuro-Psychopharmacology \& Biological Psychiatry, 31, $1044-1053$. http://dx.doi.org/10.1016/j.pnpbp.2007.03.004

[77] Gold, P.W. and Ghrousos, G.P. (2002) Organization of the Stress System and Its Dysregulation in Melancholic and Atypical Depression: High vs. Low CRH/NE States. Molecular Psychiatry, 7, 254-275. http://dx.doi.org/10.1038/sj.mp.4001032

[78] Gold, P.W., Wong, M.L., Goldstein, D.S., Gold, H.K., Ronsaville, D.S. and Esler, M. (2005) Cardiac Implications of Increased Arterial Entry and Reversible 24-h Central and Peripheral Norepinephrine Levels in Melancholia. Proceedings of the National Academy of Sciences, 102, 8303-8308. http://dx.doi.org/10.1073/pnas.0503069102

[79] Timio, M., Saronio, P., Verdura, C., Schiaroli, M., Timio, F. and Monarca, C. (2001) A Link between Psychosocial Factors and Blood Pressure Trend in Women. Physiology \& Behavior, 73, 359-363. http://dx.doi.org/10.1016/S0031-9384(01)00489-9

[80] Granath, J., Ingvarsson, S., von Thiele, U. and Lundberg, U. (2006) Stress Management: A Randomized Study of Cognitive Behavioral Therapy and Yoga. Cognitive Behavioral Therapy, 35, 3-10. http://dx.doi.org/10.1080/16506070500401292

[81] Curiati, J.A., Bocchi, E., Freire, J.O., Arantes, A.C., Braga, M., Garcia, Y., et al. (2005) Meditation Reduces Sympathetic Activation and Improves the Quality of Life in Elderly Patients with Optimally Treated Heart Failure: A Prospective Randomized Study. The Journal of Alternative and Complementary Medicine, 11, 465-472. http://dx.doi.org/10.1089/acm.2005.11.465

[82] Koenig, H.G., King, D.E. and Carson, V.B. (2012) Handbook of Religion and Health. Oxford University Press, New York. 
Scientific Research Publishing (SCIRP) is one of the largest Open Access journal publishers. It is currently publishing more than 200 open access, online, peer-reviewed journals covering a wide range of academic disciplines. SCIRP serves the worldwide academic communities and contributes to the progress and application of science with its publication.

Other selected journals from SCIRP are listed as below. Submit your manuscript to us via either submit@scirp.org or Online Submission Portal.
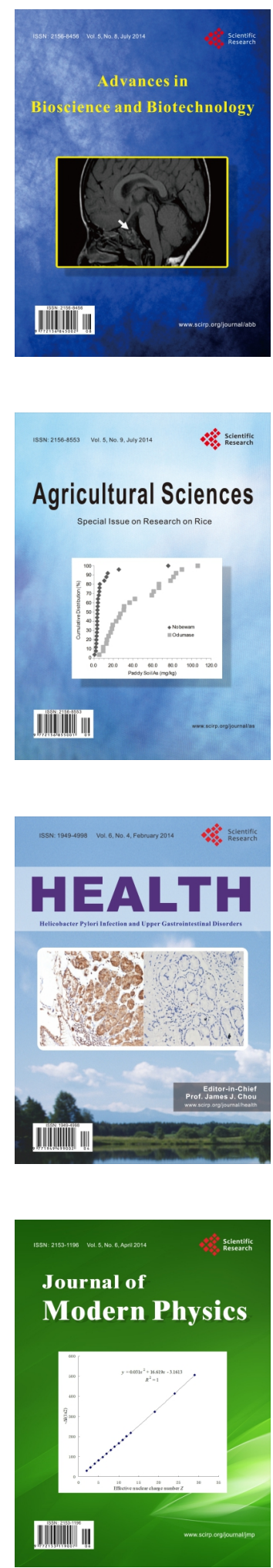
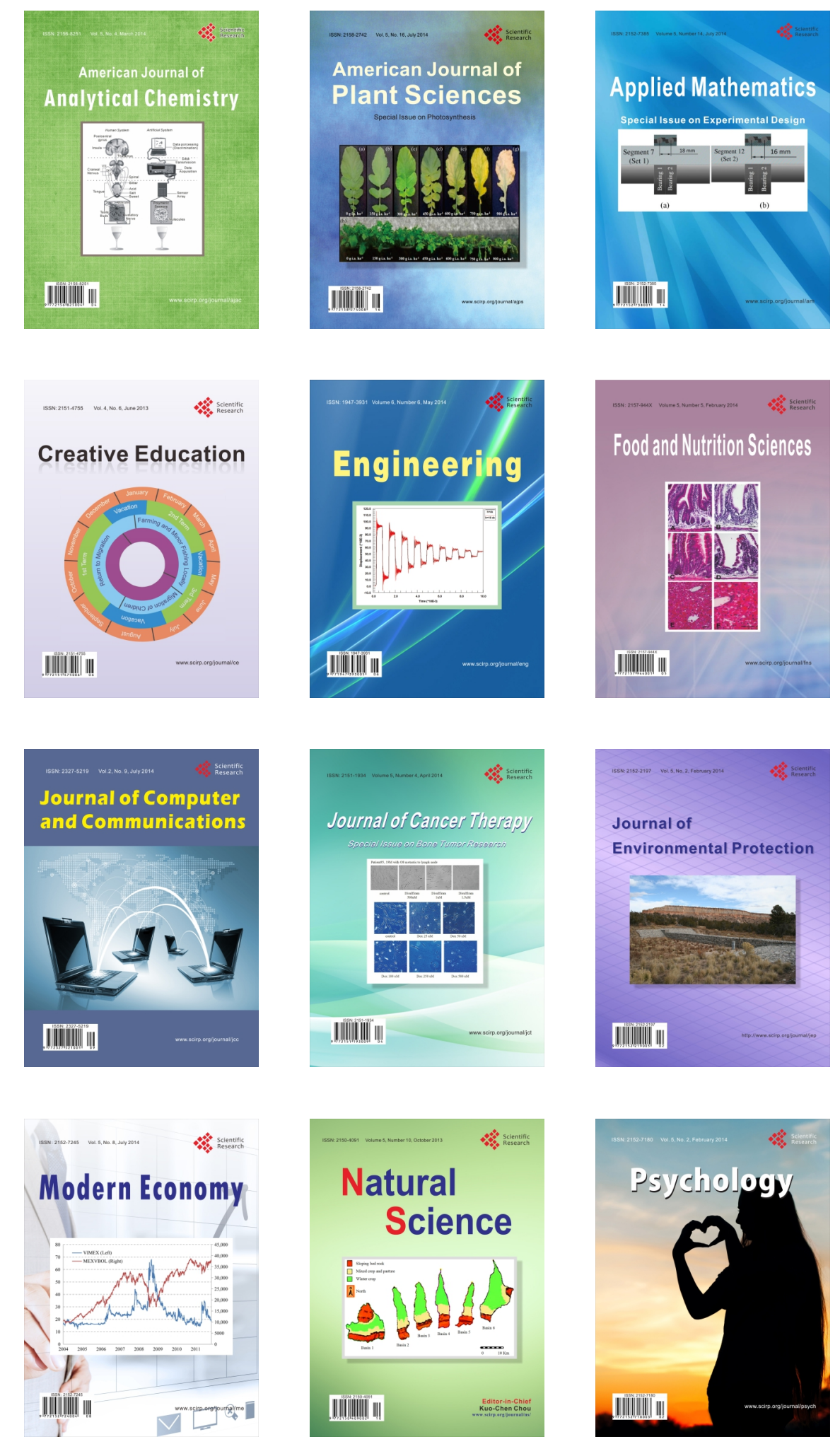